\title{
Dirac's Condition for Spanning Halin Subgraphs
}

\author{
Guantao Chen $^{\dagger}$ and Songling Shan ${ }^{\ddagger}$ \\ † Georgia State University, Atlanta, GA 30303 \\ ¥ Vanderbilt University, Nashville, TN 37240
}

July 18, 2017

\begin{abstract}
Let $G$ be an $n$-vertex graph with $n \geq 3$. A classic result of Dirac from 1952 asserts that $G$ is hamiltonian if $\delta(G) \geq n / 2$. Dirac's theorem is one of the most influential results in the study of hamiltonicity and by now there are many related known results (see, e.g., J. A. Bondy, Basic Graph Theory: Paths and Circuits, Chapter 1 in: Handbook of Combinatorics Vol.1). A Halin graph is a planar graph consisting of two edge-disjoint subgraphs: a spanning tree of at least 4 vertices and with no vertex of degree 2 , and a cycle induced on the set of the leaves of the spanning tree. Halin graphs possess rich hamiltonicity properties such as being hamiltonian, hamiltonian connected, and almost pancyclic. As a continuous "generalization" of Dirac's theorem, in this paper, we show that there exists a positive integer $n_{0}$ such that any graph $G$ with $n \geq n_{0}$ vertices and $\delta(G) \geq(n+1) / 2$ contains a spanning Halin subgraph. In particular, it contains a spanning Halin subgraph which is also pancyclic.
\end{abstract}

Keywords: Halin graph, Ladder, Dirac's condition

\section{Introduction}

A classic theorem of Dirac [11] from 1952 asserts that every graph on $n$ vertices with minimum degree at least $n / 2$ is hamiltonian if $n \geq 3$. Following Dirac's result, numerous results on hamiltonicity properties on graphs with restricted degree conditions have been obtained (see, for instance, $[14,15])$. Traditionally, under similar conditions, results for a graph being hamiltonian, hamiltonian-connected, and pancyclic are obtained separately. We may ask, under certain conditions, if it is possible to uniformly show a graph possessing several hamiltonicity properties. The work on finding the square of a hamiltonian cycle in a graph can be seen as an attempt in this direction. However, it requires minimum degree of $2 n / 3$ for an $n$-vertex graph $G$ to contain the square of a hamiltonian cycle, for examples, see $[6,12,13,19,24]$. Although the minimum degree condition of $2 n / 3$ for having the square of a hamiltonian cycle is almost optimal for the embedding result it implies: Aigner-Brandt Theorem [1] that any $n$ vertex graph with minimum degree at least $(2 n-1) / 3$ contains all possible graphs of order at most $n$ and maximum degree at most 2 , it is a "waste" for using the square of a hamiltonian cycle in obtaining 
hamiltonicity properties. For bipartite graphs, finding the existence of a spanning ladder is a way of simultaneously showing the graph having many hamiltonicity properties (see $[9,10])$. In this paper, we introduce another approach of uniformly showing the possession of several hamiltonicity properties in a graph: we show the existence of a spanning Halin graph in a graph under a given minimum degree condition.

A tree with no vertex of degree 2 is called a homeomorphically irreducible tree(HIT). A Halin graph $H=T \cup C$ is a simple planar graph consisting of a HIT $T$ with at least 4 vertices and a cycle $C$ induced by the set of leaves of $T$. The HIT $T$ is called the underlying tree of $H$. A wheel graph is an example of a Halin graph, where the underlying tree is a star. Halin constructed Halin graphs in [16] for the study of minimally 3-connected graphs. Lovász and Plummer named such graphs as Halin graphs in their study of planar bicritical graphs [20], which are planar graphs having a 1-factor after deleting any two vertices. Intensive researches have been done on Halin graphs. Bondy [4] in 1975 showed that a Halin graph is hamiltonian. In the same year, Lovász and Plummer [20] showed that not only a Halin graph itself is hamiltonian, but each of the subgraph obtained by deleting a vertex is hamiltonian. In 1987, Barefoot [3] proved that Halin graphs are hamiltonian-connected, i.e., there is a hamiltonian path connecting any two vertices of the graph. Furthermore, it was proved that each edge of a Halin graph is contained in a hamiltonian cycle and is avoided by another [23]. Bondy and Lovász [5], and Skowrońska [22], independently, in 1985, showed that a Halin graph is almost pancyclic and is pancyclic if the underlying tree has no vertex of degree 3, where an $n$-vertex graph is almost pancyclic if it contains cycles of length from 3 to $n$ with the possible exception of a single even length, and is pancyclic if it contains cycles of length from 3 to $n$. Some problems that are NP-complete for general graphs have been shown to be polynomial time solvable for Halin graphs. For example, Cornuéjols, Naddef, and Pulleyblank [8] showed that in a Halin graph, a hamiltonian cycle can be found in polynomial time. It seems so promising to show the existence of a spanning Halin subgraph in a given graph in order to show that the graph possesses many hamiltonicity properties. But, nothing comes for free, it is NP-complete to determine whether a graph contains a (spanning) Halin graph [17].

Despite all these nice properties of Halin graphs mentioned above, the problem of determining whether a graph contains a spanning Halin subgraph has not yet well studied except a conjecture proposed by Lovász and Plummer [20] in 1975. The conjecture states that every 4-connected plane triangulation contains a spanning Halin subgraph (disproved recently [7]). In this paper, we investigate the minimum degree condition for implying the existence of a spanning Halin subgraph in a graph, and thereby giving another approach for uniformly showing the possession of several hamiltonicity properties in a graph under a given minimum degree condition. We obtain the following result.

Theorem 1.1 There exists $n_{0}>0$ such that for any graph $G$ with $n \geq n_{0}$ vertices, if $\delta(G) \geq$ $(n+1) / 2$, then $G$ contains a spanning Halin subgraph. In particular, it contains a spanning Halin subgraph which is also pancyclic.

Note that an $n$-vertex graph with minimum degree at least $(n+1) / 2$ is 3-connected if $n \geq 4$. Hence, the minimum degree condition in Theorem 1.1 implies the 3 -connectedness, which is a necessary condition for a graph to contain a spanning Halin subgraph, since every Halin graph is 3-connected. A Halin graph contains a triangle, and bipartite graphs are triangle-free. Hence, $K_{\left\lfloor\frac{n}{2}\right\rfloor,\left\lceil\frac{n}{2}\right\rceil}$ contains no spanning Halin subgraph. For $n$ even, the graph obtained from two copies of $K_{\frac{n}{2}+1}$ by gluing them together on an edge is 2-connected, so it has no spanning Halin subgraph. Both these two graphs have minimum degree at most $n / 2$. We see that the 
minimum degree condition in Theorem 1.1 is best possible.

Theorem 1.1 is proved for large graphs. It might be very hard for obtaining a same result for all graphs, as when constructing a Halin graph in general, we may need to find its underlying tree first. The minimum degree condition suffices for the existence of a such tree $T$ in $G$ (in fact, it was showed that an $n$-vertex graph with minimum degree at least $4 \sqrt{2 n}$ contains a spanning tree with no vertex of degree 2 [2]). However, the hardness lies in finding a cycle $C$ spanning on the set of the leaves of $T$ so that $T \cup C$ is planar. In other words, when $T$ is fixed, we have to find a cycle $C$ in $G$ passing through a set of given vertices in some particular order. The other way of finding a spanning Halin graph $H$ is to find a spanning subgraph which contains $H$. For example, spanning structures close to ladder structures (e.g., graphs $H_{1}$ to $H_{5}$ as defined in next section). Particularly, the square of a hamiltonian cycle contains $H_{1}$ or $H_{2}$ as a spanning subgraph, so it contains a spanning Halin subgraph. But the disadvantage of using "uniform" structures as $H_{i}$ is that it makes it hard for constructing them "manually". Nevertheless, we still suspect that $(n+1) / 2$ is the right condition for all graphs to contain a spanning Halin subgraph.

\section{Notation and definitions}

We consider simple and finite graphs only. Let $G$ be a graph. Denote by $V(G)$ and $E(G)$ the vertex set and edge set of $G$, respectively, and by $e(G)$ the cardinality of $E(G)$. We denote by $\delta(G)$ the minimum degree of $G$ and by $\Delta(G)$ the maximum degree. Let $v \in V(G)$ be a vertex and $S \subseteq V(G)$ a subset. Then $G[S]$ is the subgraph of $G$ induced by $S$. Similarly, $G[F]$ is the subgraph induced by $F$ if $F \subseteq E(G)$. The notation $\Gamma_{G}(v, S)$ denotes the set of neighbors of $v$ in $S$, and $\operatorname{deg}_{G}(v, S)=\left|\Gamma_{G}(v, S)\right|$. We let $\Gamma_{\bar{G}}(v, S)=S-\Gamma_{G}(v, S)$ and $\operatorname{deg}_{\bar{G}}(v, S)=\left|\Gamma_{\bar{G}}(v, S)\right|$. Given another set $U \subseteq V(G)$, define $\Gamma_{G}(U, S)=\bigcap_{u \in U} \Gamma_{G}(u, S)$, $\operatorname{deg}_{G}(U, S)=\left|\Gamma_{G}(U, S)\right|$, and $N_{G}(U, S)=\bigcup_{u \in U} \Gamma_{G}(u, S)$. When $U=\left\{u_{1}, u_{2}, \cdots, u_{k}\right\}$, we may write $\Gamma_{G}(U, S), \operatorname{deg}_{G}(U, S)$, and $N_{G}(U, S)$ as $\Gamma_{G}\left(u_{1}, u_{2}, \cdots, u_{k}, S\right), \operatorname{deg}_{G}\left(u_{1}, u_{2}, \cdots, u_{k}, S\right)$, and $N_{G}\left(u_{1}, u_{2}, \cdots, u_{k}, S\right)$, respectively, in specifying the vertices in $U$. When $S=V(G)$, we only write $\Gamma_{G}(U), \operatorname{deg}_{G}(U)$, and $N_{G}(U)$. Let $U_{1}, U_{2} \subseteq V(G)$ be two disjoint subsets. Then $\delta_{G}\left(U_{1}, U_{2}\right)=\min \left\{\operatorname{deg}_{G}\left(u_{1}, U_{2}\right) \mid u_{1} \in U_{1}\right\}$ and $\Delta_{G}\left(U_{1}, U_{2}\right)=\max \left\{\operatorname{deg}_{G}\left(u_{1}, U_{2}\right) \mid u_{1} \in U_{1}\right\}$. Notice that the notation $\delta_{G}\left(U_{1}, U_{2}\right)$ and $\Delta_{G}\left(U_{1}, U_{2}\right)$ are not symmetric with respect to $U_{1}$ and $U_{2}$. We denote by $E_{G}\left(U_{1}, U_{2}\right)$ the set of edges with one end in $U_{1}$ and the other in $U_{2}$, the cardinality of $E_{G}\left(U_{1}, U_{2}\right)$ is denoted by $e_{G}\left(U_{1}, U_{2}\right)$. We may omit the index $G$ if there is no risk of confusion. Let $u, v \in V(G)$ be two vertices. We write $u \sim v$ if $u$ and $v$ are adjacent. A path connecting $u$ and $v$ is called a $(u, v)$-path. If $G$ is a bipartite graph with partite sets $A$ and $B$, we denote $G$ by $G(A, B)$ in emphasizing the two partite sets.

In constructing Halin graphs, we use ladder graphs and a class of "ladder-like" graphs as substructures. We give the description of these graphs below.

Definition 1 An n-ladder $L_{n}=L_{n}(A, B)$ is a balanced bipartite graph with $A=\left\{a_{1}, a_{2}, \cdots, a_{n}\right\}$ and $B=\left\{b_{1}, b_{2}, \cdots, b_{n}\right\}$ such that $a_{i} \sim b_{j}$ iff $|i-j| \leq 1$. We call $a_{i} b_{i}$ the $i$-th rung of $L_{n}$. If $2 n(\bmod 4) \equiv 0$, then we call each of the shortest $\left(a_{1}, b_{n}\right)$-path $a_{1} b_{2} a_{3} b_{4} \cdots a_{n-1} b_{n}$ and $\left(b_{1}, a_{n}\right)$ path $b_{1} a_{2} b_{3} a_{4} \cdots b_{n-1} a_{n}$ a side of $L_{n}$; and if $2 n(\bmod 4) \equiv 2$, then we call each of the shortest $\left(a_{1}, a_{n}\right)$-path $a_{1} b_{2} a_{3} b_{4} \cdots a_{n-1} b_{n-1} a_{n}$ and $\left(b_{1}, b_{n}\right)$-path $b_{1} a_{2} b_{3} a_{4} \cdots b_{n-2} a_{n-1} b_{n}$ a side of $L_{n}$.

Let $L$ be a ladder with $x y$ as one of its rungs. For an edge $g h$, we say $x y$ and $g h$ are adjacent if $x \sim g, y \sim h$ or $x \sim h, y \sim g$. Suppose $L$ has its first rung as $a b$ and its last rung as $c d$, we 
denote $L$ by $a b-L-c d$ in specifying the two rungs, and we always assume that the distance between $a$ and $c$ and thus between $b$ and $d$ is $|V(L)| / 2-1$ (we make this assumption for being convenient in constructing other graphs based on ladders). Under this assumption, we denote $L$ as $\overrightarrow{a b}-L-\overrightarrow{c d}$. Let $A$ and $B$ be two disjoint vertex sets. We say the rung $x y$ of $L$ is contained in $A \times B$ if either $x \in A, y \in B$ or $x \in B, y \in A$. Let $L^{\prime}$ be another ladder vertex-disjoint with $L$. If the last rung of $L$ is adjacent to the first rung of $L^{\prime}$, we write $L L^{\prime}$ for the new ladder obtained by concatenating $L$ and $L^{\prime}$. In particular, if $L^{\prime}=g h$ is an edge, we write $L L^{\prime}$ as $L g h$.

We now define five types of "ladder-like" graphs, call them $H_{1}, H_{2}, H_{3}, H_{4}$ and $H_{5}$, respectively. Let $L_{n}$ be a ladder with $a_{1} b_{1}$ and $a_{n} b_{n}$ as the first and last rung, respectively, and $x, y, z, w, u$ be five new vertices. Then each of $H_{i}$ is obtained from $L_{n}$ by adding some specified vertices and edges as follows. Additionally, for each $i$ with $1 \leq i \leq 5$, we define a graph $T_{i}$ associated with $H_{i}$. A depiction of a ladder $L_{4}, H_{1}, H_{2}, H_{3}, H_{4}, H_{5}$ constructed from $L_{4}$, and the graph $T_{i}$ associated with $H_{i}$ is given in Figure 1.

$H_{1}$ : Adding two new vertices $x, y$ and the edges $x a_{1}, x b_{1}, y a_{n}, y b_{n}$ and $x y$.

Let $T_{1}=H_{1}\left[\left\{x, y, a_{1}, b_{1}, a_{n}, b_{n}\right\}\right]$.

$H_{2}$ : Adding three new vertices $x, y, z$ and the edges $z a_{1}, z b_{1}, x z, x b_{1}, y a_{n}, y b_{n}$ and $x y$.

Let $T_{2}=H_{2}\left[\left\{x, y, z, a_{1}, b_{1}, a_{n}, b_{n}\right\}\right]$.

$H_{3}$ : Adding three new vertices $x, y, z$ and the edges $x a_{1}, x b_{1}, y a_{n}, y b_{n}, x z, y z$, and either $z a_{i}$ or $z b_{i}$ for some $1 \leq i \leq n$. Note that $H_{2}$ is a special case of $H_{3}$ with $i=1$ or $n$.

Let $T_{3}=H_{3}\left[\left\{x, y, z, a_{1}, b_{1}, a_{n}, b_{n}\right\}\right]$.

$H_{4}$ : Adding four new vertices $x, y, z, w$ and the edges $w a_{1}, w b_{1}, x w, x b_{1}, y a_{n}, y b_{n}, x z, y z$, and either $z a_{i}$ or $z b_{i}$ for some $1 \leq i \leq n$ such that $a_{i}$ or $b_{i}$ is a vertex on the side of $L$ which has $b_{1}$ as one end.

Let $T_{4}=H_{4}\left[\left\{x, y, z, w, a_{1}, b_{1}, a_{n}, b_{n}\right\}\right]$.

$H_{5}$ : Adding five new vertices $x, y, z, w, u$.

If $2 n(\bmod 4) \equiv 2$, adding the edges $w a_{1}, w b_{1}, x w, x b_{1}, u a_{n}, u b_{n}, y u, y b_{n}, x z, y z$, and either $z a_{i}$ or $z b_{i}$ for some $1 \leq i \leq n$ such that $a_{i}$ or $b_{i}$ is a vertex on the shortest $\left(b_{1}, b_{n}\right)$-path in $L$;

and if $2 n(\bmod 4) \equiv 0$, adding the edges $w a_{1}, w b_{1}, x w, x b_{1}, u a_{n}, u b_{n}, y u, y a_{n}, x z, y z$, and either $z a_{i}$ or $z b_{i}$ for some $1 \leq i \leq n$ such that $a_{i}$ or $b_{i}$ is a vertex on the shortest $\left(b_{1}, a_{n}\right)$ path in $L$.

The graph obtained from $H_{5}$ by deleting the vertex $z$ and adding the edge $x y$ is identical with $H_{4}$ with $i=n$.

Let $T_{5}=H_{5}\left[\left\{x, y, z, w, u, a_{1}, b_{1}, a_{n}, b_{n}\right\}\right]$.

Let $i=1,2, \cdots, 5$. Notice that each of $H_{i}$ is a Halin graph, and the graph obtained from $H_{5}$ by deleting the vertex $z$ and adding the edge $x y$ is also a Halin graph. Except $H_{1}$, each $H_{i}$ has a unique underlying tree. Notice also that $x y$ is an edge on the cycle along the leaves of any underlying tree of $H_{1}$ or $H_{2}$. For each $H_{i}$ and $T_{i}$, call $x$ the left end and $y$ the right end, and call a vertex of degree at least 3 in the underlying tree of $H_{i}$ a Halin constructible vertex. By analyzing the structure of $H_{i}$, we see that each internal vertex on a/the shortest $(x, y)$-path in $H_{i}-x y$ (for $\left.i=1,2\right)$ or $H_{i}-z$ (for $\left.i=3,4,5\right)$ is a Halin constructible vertex. Noting that any vertex in $V\left(H_{1}\right)-\{x, y\}$ can be a Halin constructible vertex. We call $a_{1} b_{1}$ the head link of $T_{i}$ and $a_{n} b_{n}$ the tail link of $T_{i}$, and for each of $T_{3}, T_{4}, T_{5}$, we call the vertex $z$ not contained in any triangles the pendent vertex. The notation of $H_{i}$ and $T_{i}$ are fixed hereafter. 
Let $T \in\left\{T_{1}, \cdots, T_{5}\right\}$ be a subgraph of a graph $G$. Suppose that $T$ has head link $a b$, tail link $c d$, and possibly the pendent vertex $z$. Suppose $G-V(T)$ contains a spanning ladder $L$ with first rung $c_{1} d_{1}$ and last rung $c_{n} d_{n}$ such that $c_{1} d_{1}$ is adjacent to $a b, c_{n} d_{n}$ is adjacent to $c d$. Additionally, if the pendent vertex $z$ of $T$ exists, then $z$ has a neighbor $z^{\prime}$, which is an internal vertex on a shortest path between the two ends of $T$ in the graph $a b L c d \cup T-z$. Then $a b L c d \cup T$ or $a b L c d \cup T \cup\left\{z z^{\prime}\right\}$ is a spanning Halin subgraph of $G$. This technique is frequently used later on in constructing a Halin graph. The following proposition gives another way of constructing a Halin graph based on $H_{1}$ and $H_{2}$.

Proposition 1 For $i=1,2$, let $G_{i} \in\left\{H_{1}, H_{2}\right\}$ with left end $x_{i}$ and right end $y_{i}$ be defined as above, and let $u_{i} \in V\left(G_{i}\right)$ be a Halin constructible vertex, then $Q:=G_{1} \cup G_{2}-\left\{x_{1} y_{1}, x_{2} y_{2}\right\} \cup$ $\left\{x_{1} x_{2}, y_{1} y_{2}, u_{1} u_{2}\right\}$ is a Halin graph spanning on $V\left(G_{1}\right) \cup V\left(G_{2}\right)$. Let $\mathcal{Q}=\left\{Q \mid G_{i} \in\left\{H_{1}, H_{2}\right\}\right\}$ be the set of all graphs $Q$ constructed in this way. Then any graph in $\mathcal{Q}$ is pancyclic.

Proof. For $i=1,2$, let $G_{i}$ be embedded in the plane, and let $T_{G_{i}}$ be a underlying plane tree of $G_{i}$. Then $T^{\prime}:=T_{G_{1}} \cup T_{G_{2}} \cup\left\{u_{1} u_{2}\right\}$ is a homeomorphically irreducible tree spanning on $V\left(G_{1}\right) \cup V\left(G_{2}\right)$. Moreover, we can draw the edge $u_{1} u_{2}$ such that $T_{G_{1}} \cup T_{G_{2}} \cup\left\{u_{1} u_{2}\right\}$ is a plane graph. Since $G_{i}\left[E\left(G_{i}\right)-E\left(T_{G_{i}}\right)-\left\{x_{i} y_{i}\right\}\right]$ is an $\left(x_{i}, y_{i}\right)$-path spanning on the set of leaves of $T_{G_{i}}$ obtained by connecting the leaves following the order determined by the embedding of $T_{G_{i}}$, we see that $G_{1}\left[E\left(G_{1}\right)-E\left(T_{G_{1}}\right)-\left\{x_{1} y_{1}\right\}\right] \cup G_{2}\left[E\left(G_{2}\right)-E\left(T_{G_{2}}\right)-\left\{x_{2} y_{2}\right\}\right] \cup\left\{x_{1} x_{2}, y_{1} y_{2}\right\}$ is a cycle spanning on the set of leaves of $T^{\prime}$ obtained by connecting the leaves following the order determined by the embedding of $T^{\prime}$. Thus $Q$ is a Halin graph.

To see the pancyclicity of graphs in $\mathcal{Q}$, suppose that $H_{1}$ has $2 n_{1}+2$ vertices and $H_{2}$ has $2 n_{2}+3$ vertices. It is easy to check that in $H_{i}$, there are $\left(x_{i}, y_{i}\right)$-paths of length from $n_{i}+1$ to $\left|V\left(H_{i}\right)\right|-1$; in $H_{1}$, there are cycles of length from 3 to $2 n_{1}+2$; and in $H_{2}$, there are cycles of length from 3 to $2 n_{2}+3$. Let $Q \in \mathcal{Q}$ such that $Q$ is constructed based on $H_{1}$ and $H_{2}$. As $x_{1} x_{2}, y_{1} y_{2} \in E(Q)$, then we see that $Q$ has all cycles of length from $n_{1}+n_{2}+4$ to $n_{1}+n_{2}+5=|V(Q)|$. Together with cycles in $H_{i}$, we know that $Q$ contains all cycles of length from 3 to $|V(Q)|$. The pancyclicity of other graphs in $\mathcal{Q}$ can be checked similarly.
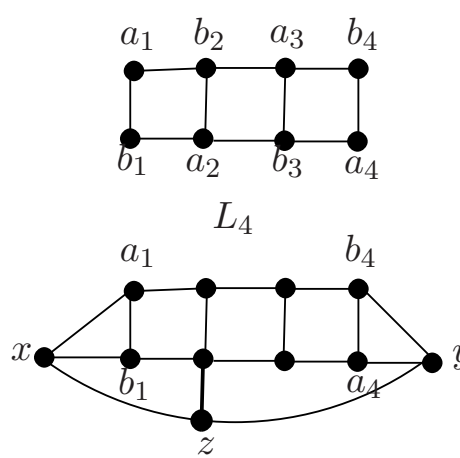

$H_{3}$

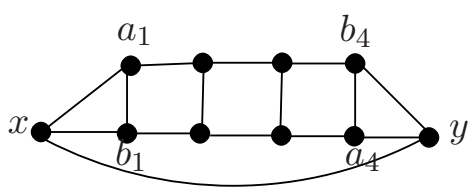

$H_{1}$

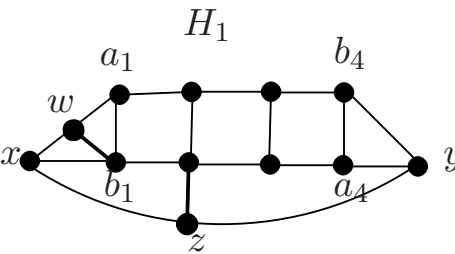

$\mathrm{H}_{4}$

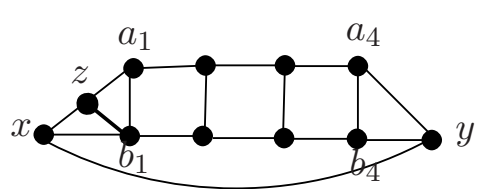

$\mathrm{H}_{2}$

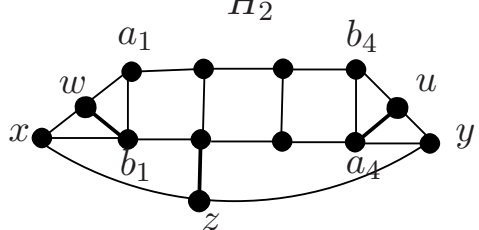

$H_{5}$

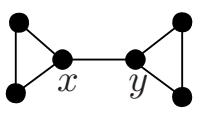

$T_{1}$

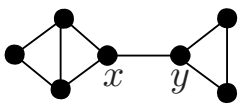

$T_{2}$

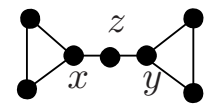

$T_{3}$

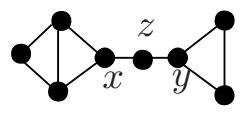

$T_{4}$

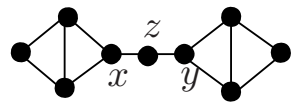

$T_{5}$

Figure 1: $L_{4}, H_{i}$ constructed from $L_{4}$, and $T_{i}$ associated with $H_{i}$ for each $i=1,2, \cdots, 5$ 


\section{Proof of Theorem 1.1}

In this section, we prove Theorem 1.1. Following the standard setup of proofs applying the Regularity Lemma, we divide the proof into Non-extremal Case and extremal cases. For this purpose, we define the two extremal cases in the following.

Let $G$ be an $n$-vertex graph and $V$ its vertex set. Let $0<\beta \leq 1$ be a constant. Let $W \subseteq V(G)$. We say $W$ is an approximate vertex-cut of $G$ with parameter $\beta$ if there is a partition $V_{1}$ and $V_{2}$ of $V-W$ such that $e_{G}\left(V_{1}, V_{2}\right) \leq \beta n^{2}$ and $\delta\left[G\left[V_{i}\right]\right] \geq \delta(G)-|W|-\beta n$ for each $i=1,2$. The two extremal cases are defined as below.

Extremal Case 1. $G$ has an approximate vertex-cut of size at most $5 \beta n$ with parameter $\beta$.

Extremal Case 2. There exists a partition $V_{1} \cup V_{2}$ of $V$ such that $\left|V_{1}\right| \geq(1 / 2-7 \beta) n$ and $\Delta\left(G\left[V_{1}\right]\right) \leq \beta n$.

Non-extremal Case. We say that an $n$-vertex graph with minimum degree at least $(n+1) / 2$ is in Non-extremal Case if it is in neither Extremal Case 1 nor Extremal Case 2.

In Extremal Case 1, we will show that $G$ contains a spanning Halin subgraph isomorphic to a graph in $\mathcal{Q}$ (defined in Proposition 1). In all other cases, we will construct a spanning subgraph of $G$ isomorphic to $H_{i}$ for some $i \in\{1,2,3,4,5\}$. Note that each graph in $\mathcal{Q}$ and each $H_{i}$ is pancyclic. Hence, to prove Theorem 1.1, we only need to show the existence of the mentioned graphs above. The following three theorems deal with the Non-extremal Case and the two extremal cases, respectively, and thus give a proof of Theorem 1.1.

Theorem 3.1 Suppose that $0<\beta \ll 1 /\left(20 \cdot 17^{3}\right)$ and $n$ is a sufficiently large integer. Let $G$ be a graph on $n$ vertices with $\delta(G) \geq(n+1) / 2$. If $G$ is in Extremal Case 1, then $G$ contains a spanning Halin isomorphic to a graph in $\mathcal{Q}$ (defined in Proposition 1) as a subgraph.

Theorem 3.2 Suppose that $0<\beta \ll 1 /\left(20 \cdot 17^{3}\right)$ and $n$ is a sufficiently large integer. Let $G$ be a graph on $n$ vertices with $\delta(G) \geq(n+1) / 2$. If $G$ is in Extremal Case 2, then $G$ contains a spanning Halin subgraph isomorphic to some $H_{i}, i \in\{1,2,3,4,5\}$.

Theorem 3.3 Let $n$ be a sufficiently large integer and $G$ an $n$-vertex graph with $\delta(G) \geq(n+$ 1)/2. If $G$ is in the Non-extremal Case, then $G$ has a spanning Halin subgraph isomorphic to $H_{1}$ or $H_{2}$.

We need the following lemma in each of the proofs of Theorems $3.1-3.2$ in dealing with "garbage" vertices.

Lemma 3.1 Let $F$ be a graph such that $V(F)$ is partitioned as $S \cup R$. Suppose that (i) there are $|R|$ vertex-disjoint 3-stars (a 3-star is a copy of $K_{1,3}$ ) with the vertices in $R$ as their centers, (ii) for any two vertices $u, v \in N(R, S)$, $\operatorname{deg}(u, v, S) \geq 6|R|$, and (iii) for any three vertices $u, v, w \in N(N(R, S), S), \operatorname{deg}(u, v, w, S) \geq 7|R|$. Then there is a ladder spanning on $R$ and some other $7|R|-2$ vertices from $S$. Particularly, the ladder has the vertices on its first and last rungs in $S$.

Proof. Let $R=\left\{w_{1}, w_{2}, \cdots, w_{r}\right\}$. Consider first that $r=1$. Choose $x_{11}, x_{12}, x_{13} \in$ $\Gamma\left(w_{1}, S\right)$. By (ii), there are distinct vertices $y_{12}^{1} \in \Gamma\left(x_{11}, x_{12}, S\right)$ and $y_{23}^{1} \in \Gamma\left(x_{12}, x_{13}, S\right)$. Then the graph $L$ on $\left\{w_{1}, x_{11}, x_{12}, x_{13}, y_{12}^{1}, y_{23}^{1}\right\}$ with edges in

$$
\left\{w_{1} x_{11}, w_{1} x_{12}, w_{1} x_{13}, y_{12}^{1} x_{11}, y_{12}^{1} x_{12}, y_{23}^{1} x_{12}, y_{23}^{1} x_{13}\right\}
$$

is a ladder covering $R$ with $|V(L)|=6$. Suppose now $r \geq 2$. By condition (i), for each $i$ with $1 \leq i \leq r$, there exist distinct vertices $x_{i 1}, x_{i 2}, x_{i 3} \in \Gamma\left(w_{i}, S\right)$. By (ii), we choose 
distinct vertices $y_{12}^{1}, y_{23}^{1}, \cdots, y_{12}^{r}, y_{23}^{r}$ different from the existing vertices already chosen such that $y_{12}^{i} \in \Gamma\left(x_{i 1}, x_{i 2}, S\right)$ and $y_{23}^{i} \in \Gamma\left(x_{i 2}, x_{i 3}, S\right)$ for each $i$, and at the same time, we chose distinct vertices $z_{1}, z_{2}, \cdots, z_{r-1}$ from the unchosen vertices in $S$ such that $z_{i} \in \Gamma\left(x_{i 3}, x_{(i+1), 1}, S\right)$ for each $1 \leq i \leq r-1$. Finally, by (iii), choose distinct vertices $u_{1}, u_{2}, \cdots, u_{r-1}$ from the unchosen vertices in $S$ such that $u_{i} \in \Gamma\left(y_{23}^{i}, y_{12}^{i+1}, z_{i}, S\right)$. Let $L$ be the graph with

$$
V(L)=R \cup\left\{x_{i 1}, x_{i 2}, x_{i 3}, y_{12}^{i}, y_{23}^{i}, z_{i}, u_{i}, x_{r 1}, x_{r 2}, x_{r 3}, y_{12}^{r}, y_{23}^{r} \mid 1 \leq i \leq r-1\right\} \quad \text { and }
$$

$E(L)$ consisting of the edges $w_{r} x_{r 1}, w_{r} x_{r 2}, w_{r} x_{r 3}, y_{12}^{r} x_{r 1}, y_{12}^{r} x_{r 2}, y_{23}^{r} x_{r 2}, y_{23}^{r} x_{r 3}$ and the edges indicated below for each $1 \leq i \leq r-1$ :

$$
w_{i} \sim x_{i 1}, x_{i 2}, x_{i 3} ; y_{12}^{i} \sim x_{i 1}, x_{i 2} ; y_{23}^{i} \sim x_{i 2}, x_{i 3} ; z_{i} \sim x_{i 3}, x_{i+1,1} ; u_{i} \sim x_{i 3}, x_{i+1,1}, z_{i} .
$$

It is easy to check that $L$ is a ladder covering $R$ with $|V(L)|=8 r-2$. The ladder has its first and last rungs in $S$ is seen by its construction. Figure 2 gives a depiction of $L$ for $|R|=2$.

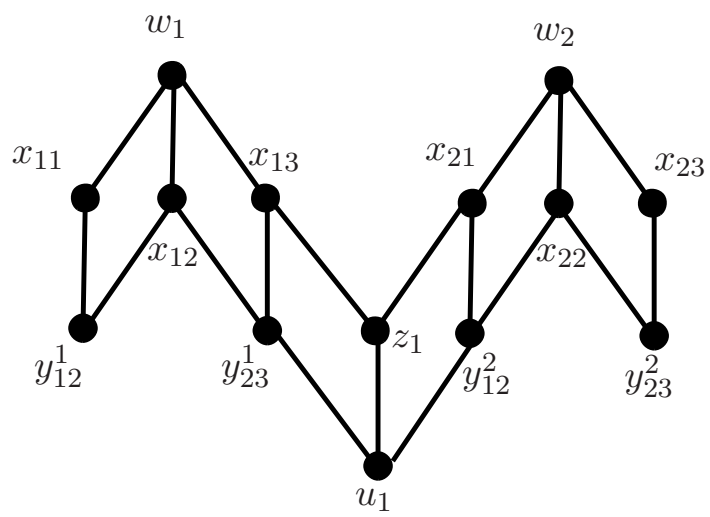

Figure 2: Ladder $L$ of order 14

We will also need the bipartite version of Lemma 3.1. Since the proof is similar, we omit it.

Lemma 3.2 Let $F(A, B)$ be a bipartite graph such that $V(F)$ is partitioned as $S \cup R$ with $R \subseteq A$. Suppose that (i) there are $|R|$ vertex-disjoint 3-stars with the vertices in $R$ as their centers, (ii) for any two vertices $u, v \in N(R, S)$, $\operatorname{deg}(u, v, S) \geq 3|R|$, and (iii) for any three vertices $u, v, w \in N(N(R, S), S), \operatorname{deg}(u, v, w, S) \geq 4|R|$. Then there is a ladder spanning on $R$ and some other $7|R|-2$ vertices from $S$ with $3|R|-1$ of them taken from $A$. Particularly, the ladder has its first and last rungs in $S$.

The following simple observation is heavily used in the proofs explicitly or implicitly.

Lemma 3.3 Let $U=\left\{u_{1}, u_{2} \cdots, u_{k}\right\}, S \subseteq V(G)$ be subsets. Then $\operatorname{deg}\left(u_{1}, u_{2}, \cdots, u_{k}, S\right) \geq$ $|S|-\left(\operatorname{deg}_{\bar{G}}\left(u_{1}, S\right)+\cdots+\operatorname{deg}_{\bar{G}}\left(u_{k}, S\right)\right) \geq|S|-k(|S|-\delta(U, S))=k \delta(U, S)-(k-1)|S|$.

Extremal Case 1 is easier than the other cases, so we start with it.

\subsection{Proof of Theorem 3.1}

We assume that $G$ has an approximate vertex-cut $W$ with parameter $\beta$ such that $|W| \leq 5 \beta n$. Let $V_{1}$ and $V_{2}$ be the partition of $V-W$ such that $\delta\left[G\left[V_{i}\right]\right] \geq(1 / 2-6 \beta) n$. As $\delta(G) \geq(n+1) / 2$, $(1 / 2-6 \beta) n \leq\left|V_{i}\right| \leq(1 / 2+6 \beta) n$. We partition $W$ into two subsets as follows:

$$
W_{1}=\left\{w \in W \mid \operatorname{deg}\left(w, V_{1}\right) \geq(n+1) / 4-2.5 \beta n\right\} \quad \text { and } \quad W_{2}=W-W_{1} .
$$


As $\delta(G) \geq(n+1) / 2$, we have $\operatorname{deg}\left(w, V_{2}\right) \geq(n+1) / 4-2.5 \beta n$ for any $w \in W_{2}$. Since $G$ is 3 connected and $(1 / 2-6 \beta) n>3$, there are three independent edges $p_{1} p_{2}, q_{1} q_{2}$, and $r_{1} r_{2}$ between $G\left[V_{1} \cup W_{1}\right]$ and $G\left[V_{2} \cup W_{2}\right]$ with $p_{1}, q_{1}, r_{1} \in V_{1} \cup W_{1}$ and $p_{2}, q_{2}, r_{2} \in V_{2} \cup W_{2}$.

For $i=1,2$, by the partition of $W_{i}$, we see that $\delta\left(W_{i}, V_{i}\right) \geq 3\left|W_{i}\right|+3$. Thus, $\delta\left(W_{i}, V_{i}-\right.$ $\left.\left\{p_{i}, q_{i}\right\}\right) \geq 3\left|W_{i}\right|$. There are $\left|W_{i}-\left\{p_{i}, q_{i}\right\}\right|$ vertex-disjoint 3 -stars with their centers in $W_{i}-$ $\left\{p_{i}, q_{i}\right\}$. By Lemma 3.3, we have

$$
\begin{aligned}
\operatorname{deg}\left(u, v, V_{i}-\left\{p_{i}, q_{i}\right\}\right) & \geq 2 \delta\left(G\left[V_{i}\right]\right)-\left|V_{i}\right| \geq(1 / 2-18 \beta) n \geq 6\left|W_{i}\right|, \quad \text { for any } u, v \in V_{i} ; \\
\operatorname{deg}\left(u, v, w, V_{i}-\left\{p_{i}, q_{i}\right\}\right) & \geq 3 \delta\left(G\left[V_{i}\right]\right)-2\left|V_{i}\right| \geq(1 / 2-30 \beta) n \geq 7\left|W_{i}\right|, \quad \text { for any } u, v, w \in V_{i} .
\end{aligned}
$$

By Lemma 3.1, we can find a ladder $L_{i}$ which spans $W_{i}-\left\{p_{i}, q_{i}\right\}$ and another $7\left|W_{i}-\left\{p_{i}, q_{i}\right\}\right|-2$ vertices from $V_{i}-\left\{p_{i}, q_{i}\right\}$, if $W_{i}-\left\{p_{i}, q_{i}\right\} \neq \emptyset$. Denote $a_{i} b_{i}$ and $c_{i} d_{i}$ the first and last rung of $L_{i}$ (if $L_{i}$ exists), respectively. Let

$$
G_{i}=G\left[V_{i}-V\left(L_{i}\right)\right] \quad \text { and } \quad n_{i}=\left|V\left(G_{i}\right)\right| .
$$

Then for $i=1,2$, if $x \in V\left(G_{i}\right)$ and $x \notin\left\{p_{i}, q_{i}\right\} \cap W$,

$n_{i} \geq(n+1) / 2-6 \beta n-7\left|W_{i}\right| \geq(n+1) / 2-41 \beta n, \quad \operatorname{deg}_{G_{i}}(x) \geq \delta\left(G\left[V_{i}\right]\right)-7\left|W_{i}\right| \geq(n+1) / 2-41 \beta n$.

If $p_{i} \in W$, then $\operatorname{deg}_{G_{i}}\left(p_{i}\right) \geq(n+1) / 4-2.5 \beta n-7\left|W_{i}\right| \geq(1 / 4-41 \beta) n$. Similarly, if $q_{i} \in W$, then $\operatorname{deg}_{G_{i}}\left(q_{i}\right) \geq(1 / 4-41 \beta) n$.

Let $i=1,2$. We now show that $G_{i}$ contains a spanning subgraph isomorphic to either $H_{1}$ or $H_{2}$ as defined in the previous section. Since $n_{i} \leq(1 / 2+6 \beta) n$ and $\operatorname{deg}_{G_{i}}(x) \geq(n+1) / 2-41 \beta n$ for any $x \in V\left(G_{i}\right)-W$, any subgraph of $G_{i}$ induced by at least $(1 / 4-41 \beta) n$ vertices not in $W$ has minimum degree at least $(n+1) / 2-41 \beta n-\left(n_{i}-(1 / 4-41 \beta) n\right) \geq(1 / 4-88 \beta) n$, and thus has a matching of size at least 2 . Hence, when $n_{i}$ is even, we can choose independent edges $e_{i}=x_{i} y_{i}$ and $f_{i}=z_{i} w_{i}$ with

$$
x_{i}, y_{i} \in \Gamma_{G_{i}}\left(p_{i}\right)-\left\{q_{i}\right\} \quad \text { and } \quad z_{i}, w_{i} \in \Gamma_{G_{i}}\left(q_{i}\right)-\left\{p_{i}\right\} .
$$

And if $n_{i}$ is odd, we can choose independent edges $g_{i} y_{i}$ (we may assume $g_{i} \neq r_{i}$ ), $f_{i}=z_{i} w_{i}$, and a vertex $x_{i}$ with

$$
g_{i}, x_{i}, y_{i} \in \Gamma_{G_{i}}\left(p_{i}\right)-\left\{q_{i}\right\}, x_{i} \in \Gamma_{G_{i}}\left(g_{i}, y_{i}\right)-\left\{p_{i}, q_{i}\right\} \quad \text { and } \quad z_{i}, w_{i} \in \Gamma_{G_{i}}\left(q_{i}\right)-\left\{x_{i}, p_{i}\right\},
$$

where the existence of the vertex $x_{i}$ is possible since the subgraph of $G_{i}$ induced by $\Gamma_{G_{i}}\left(p_{i}\right)$ has minimum degree at least $(1 / 2-41 \beta) n-\left((1 / 2+6 \beta) n-\left|\Gamma_{G_{i}}\left(p_{i}\right)\right|\right) \geq\left|\Gamma_{G_{i}}\left(p_{i}\right)\right|-47 \beta n$, and hence contains a triangle. In this case, again, denote $e_{i}=x_{i} y_{i}$. Let

$$
\begin{cases}G_{i}^{\prime}=G_{i}-\left\{p_{i}, q_{i}\right\}, & \text { if } n_{i} \text { is even; } \\ G_{i}^{\prime}=G_{i}-\left\{p_{i}, q_{i}, g_{i}\right\}, & \text { if } n_{i} \text { is odd. }\end{cases}
$$

By the definition above, $\left|V\left(G_{i}^{\prime}\right)\right|$ is even.

The following claim is a modification of (1) of Lemma 2.2 in [10].

Claim 3.1.1 For $i=1,2$, let $a_{i}^{\prime} b_{i}^{\prime}, c_{i}^{\prime} d_{i}^{\prime} \in E\left(G_{i}^{\prime}\right)$ be two independent edges. Then $G_{i}^{\prime}$ contains two vertex disjoint ladders $Q_{i 1}$ and $Q_{i 2}$ spanning on $V\left(G_{i}^{\prime}\right)$ such that $Q_{i 1}$ has $e_{i}=x_{i} y_{i}$ as its first rung, $a_{i}^{\prime} b_{i}^{\prime}$ as its last rung, and $Q_{i 2}$ has $c_{i}^{\prime} d_{i}^{\prime}$ as its first rung and $f_{i}=z_{i} w_{i}$ as its last rung, where $e_{i}$ and $f_{i}$ are defined prior to this claim. 
Proof. We only show the claim for $i=1$ as the case for $i=2$ is similar. Notice that by the definition of $G_{1}^{\prime},\left|V\left(G_{1}^{\prime}\right)\right|$ is even. Since $\left|V\left(G_{1}^{\prime}\right)\right| \leq(1 / 2+6 \beta) n$ and $\delta\left(G_{1}^{\prime}\right) \geq(n+1) / 2-41 \beta n-$ $3 \geq\left|V\left(G_{1}^{\prime}\right)\right| / 2+4, G_{1}^{\prime}$ has a perfect matching $M$ containing $e_{1}, f_{1}, a_{1}^{\prime} b_{1}^{\prime}, c_{1}^{\prime} d_{1}^{\prime}$. We identify $a_{1}^{\prime}$ and $c_{1}^{\prime}$ into a vertex called $s^{\prime}$, and identify $b_{1}^{\prime}$ and $d_{1}^{\prime}$ into a vertex called $t^{\prime}$. Denote $G_{1}^{\prime \prime}$ as the resulting graph. Note that $s^{\prime} t^{\prime} \in E\left(G_{1}^{\prime \prime}\right)$ by the way of identifications. Partition $V\left(G_{1}^{\prime \prime}\right)$ arbitrarily into $U_{1}$ and $U_{2}$ with $\left|U_{1}\right|=\left|U_{2}\right|$ such that $x_{1}, z_{1}, s^{\prime} \in U_{1}, y_{1}, w_{1}, t^{\prime} \in U_{2}$, and let $M^{\prime}:=\left(M-\left\{a_{1}^{\prime} b_{1}^{\prime}, c_{1}^{\prime} d_{1}^{\prime}\right\}\right) \cup\left\{s^{\prime} t^{\prime}\right\} \subseteq E_{G_{1}^{\prime \prime}}\left(U_{1}, U_{2}\right)$. Define an auxiliary graph $H^{\prime}$ with vertex set $M^{\prime}$ and edge set defined as follows. If $x y, u v \in M^{\prime}-\left\{s^{\prime} t^{\prime}\right\}$ with $x, u \in U_{1}$ then $x y \sim_{H^{\prime}} u v$ if and only if $x \sim_{G_{1}^{\prime}} v$ and $y \sim_{G_{1}^{\prime}} u$ (we do not include the case that $x \sim_{G_{1}^{\prime}} u$ and $y \sim_{G_{1}^{\prime}} v$ as we defined a bipartition here). Particularly, for any $p q \in M^{\prime}-\left\{s^{\prime} t^{\prime}\right\}$ with $p \in U_{1}, p q \sim_{H^{\prime}} s^{\prime} t^{\prime}$ if and only if $p \sim_{G_{1}^{\prime}} b_{1}^{\prime}, d_{1}^{\prime}$ and $q \sim_{G_{1}^{\prime}} a_{1}^{\prime}, c_{1}^{\prime}$. Notice that a ladder with rungs in $M^{\prime}$ is corresponding to a path in $H^{\prime}$ and vice versa. Since $(1 / 2-41 \beta) n-3 \leq\left|V\left(G_{1}^{\prime}\right)\right| \leq(1 / 2+6 \beta) n-2$ and $\delta\left(G_{1}^{\prime}\right) \geq$ $(n+1) / 2-41 \beta n-3$, any two vertices in $G_{1}^{\prime}$ has at least $(1 / 2-88 \beta) n-4 \geq(1 / 2-89 \beta) n$ common neighbors by Lemma 3.3. This together with the fact that $\left|U_{1}\right|=\left|U_{2}\right| \leq\left|V\left(G_{1}^{\prime \prime}\right)\right| / 2 \leq(1 / 4+3 \beta) n$ gives that $\delta\left(U_{1}, U_{2}\right), \delta\left(U_{2}, U_{1}\right) \geq(1 / 4-92 \beta) n$. For each edge $u v \in M^{\prime}$ with $u \in U_{1}, u$ is adjacent to at least $(1 / 4-92 \beta) n$ other vertices in $U_{2}$ saturated by $M^{\prime}$. Thus there are at least $(1 / 4-92 \beta) n$ edges $\left\{u_{j} v_{j} \mid v_{j} \in \Gamma_{G}\left(u, U_{2}\right)\right\} \subseteq M^{\prime}$. Among these vertices $\left\{u_{j} \mid v_{j} \in \Gamma_{G}\left(u, U_{2}\right)\right\}$ in $U_{1}$, at least $(1 / 4-92 \beta) n-((1 / 4+3 \beta) n-(1 / 4-92 \beta) n)=(1 / 4-187 \beta) n$ of them are neighbors of $v$. Thus, in $H^{\prime}, u v$ is adjacent to at least $(1 / 4-187 \beta) n$ neighbors, and thus

$$
\delta\left(H^{\prime}\right) \geq(1 / 4-187 \beta) n \geq\left|V\left(H^{\prime}\right)\right| / 2+1,
$$

since $\beta<1 / 2200$ and $n$ is very large. Hence $H^{\prime}$ has a hamiltonian path starting with $e_{1}$, ending with $f_{1}$, and having $s^{\prime} t^{\prime}$ as an internal vertex. The path with $s^{\prime} t^{\prime}$ replaced by $a_{1}^{\prime} b_{1}^{\prime}$ and $c_{1}^{\prime} d_{1}^{\prime}$ is corresponding to the required ladders in $G_{1}^{\prime}$.

We may assume $n_{1}$ is even and $n_{2}$ is odd and construct a spanning Halin subgraph of $G$ (the construction for the other three cases follow a similar argument). Recall that $p_{1} p_{2}, q_{1} q_{2}, r_{1} r_{2}$ are the three prescribed independent edges between $G\left[V_{1} \cup W_{1}\right]$ and $G\left[V_{2} \cup W_{2}\right]$, where $p_{1}, q_{1}, r_{1} \in V_{1} \cup$ $W_{1}$ and $p_{2}, q_{2}, g_{2}, r_{2} \in V_{2} \cup W_{2}$. For a uniform discussion, we may assume that both of the ladders $L_{1}$ and $L_{2}$ exist. Let $i=1,2$. Recall that $L_{i}$ has $a_{i} b_{i}$ as its first rung and $c_{i} d_{i}$ as its last rung. Choose $a_{i}^{\prime} \in \Gamma_{G}\left(a_{i}, V\left(G_{i}^{\prime}\right)\right), b_{i}^{\prime} \in \Gamma_{G}\left(b_{i}, V\left(G_{i}^{\prime}\right)\right)$ such that $a_{i}^{\prime} b_{i}^{\prime} \in E(G)$ and $c_{i}^{\prime} \in \Gamma_{G}\left(c_{i}, V\left(G_{i}^{\prime}\right)\right)$, $d_{i}^{\prime} \in \Gamma_{G}\left(d_{i}, V\left(G_{i}^{\prime}\right)\right)$ such that $c_{i}^{\prime} d_{i}^{\prime} \in E(G)\left(a_{i}^{\prime}, b_{i}^{\prime}, c_{i}^{\prime}, d_{i}^{\prime}\right.$ are chosen mutually distinct and distinct from $\left.x_{i}, y_{i}, z_{i}, w_{i}, g_{i}, r_{i}\right)$. This is possible as $\delta\left(V_{i}, V\left(G_{i}^{\prime}\right)\right) \geq(n+1) / 2-41 \beta n-2$. Let $Q_{i 1}$ and $Q_{i 2}$ be the ladders of $G_{i}^{\prime}$ given by Claim 3.1.1. Set $H_{a}=Q_{11} L_{1} Q_{12} \cup\left\{p_{1} x_{1}, p_{1} y_{1}, q_{1} z_{1}, q_{1} w_{1}\right\}$. Assume $Q_{21} L_{2} Q_{22}$ is a ladder can be denoted as $\overrightarrow{x_{2} y_{2}}-Q_{21} L_{2} Q_{22}-\overrightarrow{z_{2} w_{2}}$. To make $r_{2}$ a Halin constructible vertex, we let $H_{b}=Q_{21} L_{2} Q_{22} \cup\left\{g_{2} x_{2}, g_{2} y_{2}, p_{2} g_{2}, p_{2} y_{2}, q_{2} z_{2}, q_{2} w_{2}\right\}$ if $r_{2}$ is on the shortest $\left(y_{2}, w_{2}\right)$-path in $Q_{21} L_{2} Q_{22}$, and let $H_{b}=Q_{21} L_{2} Q_{22} \cup\left\{g_{2} x_{2}, g_{2} y_{2}, p_{2} g_{2}, p_{2} x_{2}, q_{2} z_{2}, q_{2} w_{2}\right\}$ if $r_{2}$ is on the shortest $\left(x_{2}, z_{2}\right)$-path (recall that $g_{2}, x_{2}, y_{2} \in \Gamma_{G_{2}}\left(p_{2}\right)$ ). Let $H=H_{a} \cup H_{b} \cup$ $\left\{p_{1} p_{2}, r_{1} r_{2}, q_{1} q_{2}\right\}$. Then $H$ is a spanning Halin subgraph of $G$ by Proposition 1 as $H_{a} \cup p_{1} q_{1} \cong H_{1}$ and $H_{b} \cup p_{2} q_{2} \cong H_{2}$. Figure 3 gives a construction of $H$ for the above case when $r_{2}$ is on the shortest $\left(y_{2}, w_{2}\right)$-path in $Q_{21} L_{2} Q_{22}$.

\subsection{Proof of Theorem 3.2}

Recall Extremal Case 2: There exists a partition $V_{1} \cup V_{2}$ of $V$ such that $\left|V_{1}\right| \geq(1 / 2-7 \beta) n$ and $\Delta\left(G\left[V_{1}\right]\right) \leq \beta n$. Since $\delta(G) \geq(n+1) / 2$, the assumptions imply that

$$
(1 / 2-7 \beta) n \leq\left|V_{1}\right| \leq(1 / 2+\beta) n \quad \text { and } \quad(1 / 2-\beta) n \leq\left|V_{2}\right| \leq(1 / 2+7 \beta) n .
$$




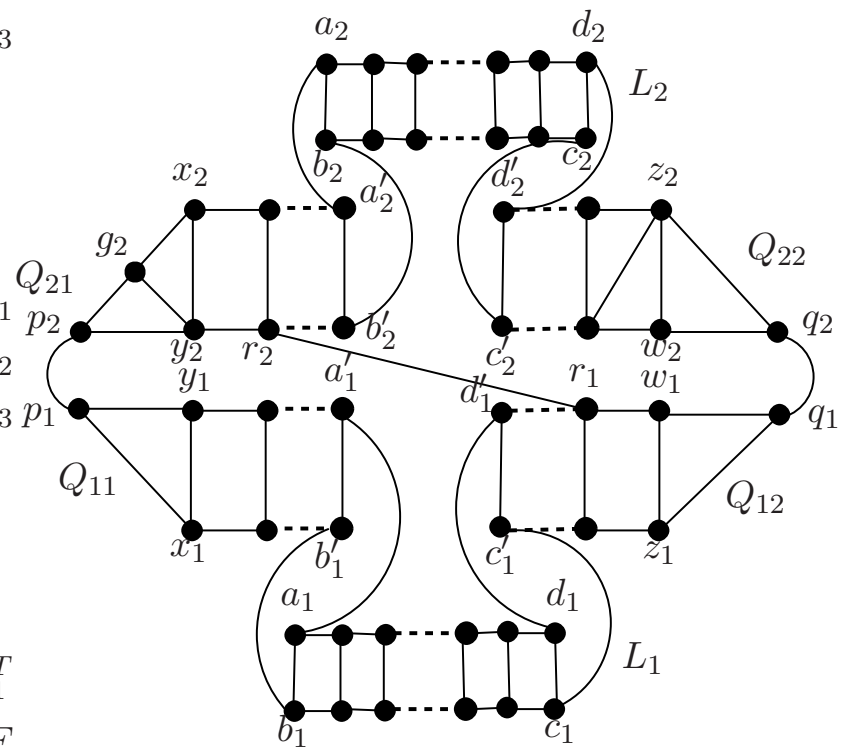

Figure 3: A Halin graph $H$

Let $\beta$ and $\alpha$ be real numbers satisfying $\beta \leq \alpha / 20$ and $\alpha \leq(1 / 17)^{3}$. Set $\alpha_{1}=\alpha^{1 / 3}$ and $\alpha_{2}=\alpha^{2 / 3}$. We first repartition $V(G)$ as follows.

$$
\begin{aligned}
& V_{2}^{\prime}=\left\{v \in V_{2}\left|\operatorname{deg}\left(v, V_{1}\right) \geq\left(1-\alpha_{1}\right)\right| V_{1} \mid\right\}, V_{01}=\left\{v \in V_{2}-V_{2}^{\prime}\left|\operatorname{deg}\left(v, V_{2}^{\prime}\right) \geq\left(1-\alpha_{1}\right)\right| V_{2}^{\prime} \mid\right\}, \\
& V_{1}^{\prime}=V_{1} \cup V_{01}, \quad \text { and } V_{0}=V_{2}-V_{2}^{\prime}-V_{01} .
\end{aligned}
$$

Claim 3.2.1 $\left|V_{01} \cup V_{0}\right|=\left|V_{2}-V_{2}^{\prime}\right| \leq \alpha_{2}\left|V_{2}\right|$.

Proof. Notice that $e\left(V_{1}, V_{2}\right) \geq(1 / 2-\beta) n\left|V_{1}\right| \geq \frac{1 / 2-\beta}{1 / 2+7 \beta}\left|V_{1}\right|\left|V_{2}\right| \geq(1-\alpha)\left|V_{1}\right|\left|V_{2}\right|$ as $\beta \leq$ $\alpha / 20$. Hence,

$$
(1-\alpha)\left|V_{1}\right|\left|V_{2}\right| \leq e\left(V_{1}, V_{2}\right) \leq e\left(V_{1}, V_{2}^{\prime}\right)+e\left(V_{1}, V_{2}-V_{2}^{\prime}\right) \leq\left|V_{1}\right|\left|V_{2}^{\prime}\right|+\left(1-\alpha_{1}\right)\left|V_{1}\right|\left|V_{2}-V_{2}^{\prime}\right| .
$$

This gives that $\left|V_{01} \cup V_{0}\right|=\left|V_{2}-V_{2}^{\prime}\right| \leq \alpha_{2}\left|V_{2}\right|$.

As a result of moving vertices from $V_{2}$ to $V_{1}$ and by Claim 3.2.1, we have the following.

$$
\begin{aligned}
(1 / 2-7 \beta) n \leq\left|V_{1}^{\prime}\right| & \leq(1 / 2+\beta) n+\left|V_{01}\right| \leq(1 / 2+\beta) n+\alpha_{2}(1 / 2+7 \beta) n \leq\left(1 / 2+\alpha_{2}\right) n, \\
\left(1 / 2-\alpha_{2}\right) n \leq\left|V_{2}^{\prime}\right| & \leq(1 / 2+7 \beta) n, \\
\delta\left(V_{1}^{\prime}, V_{2}^{\prime}\right) & \geq \min \left\{(1 / 2-\beta) n-\left|V_{2}-V_{2}^{\prime}\right|,\left(1-\alpha_{1}\right)\left|V_{2}^{\prime}\right|\right\} \geq\left(1 / 2-2 \alpha_{1} / 3\right) n, \\
\delta\left(V_{2}^{\prime}, V_{1}^{\prime}\right) & \geq\left(1-\alpha_{1}\right)\left|V_{1}\right| \geq\left(1-\alpha_{1}\right)(1 / 2-7 \beta) n \geq\left(1 / 2-2 \alpha_{1} / 3\right) n, \\
\delta\left(V_{0}, V_{1}^{\prime}\right) & \geq(n+1) / 2-\left(1-\alpha_{1}\right)\left|V_{2}^{\prime}\right|-\left|V_{0}\right| \geq \alpha_{1} n / 3 \geq 6\left|V_{0}\right|+20, \\
\delta\left(V_{0}, V_{2}^{\prime}\right) & \geq(n+1) / 2-\left(1-\alpha_{1}\right)\left|V_{1}\right|-\left|V_{0} \cup V_{01}\right| \geq \alpha_{1} n / 3 \geq 6\left|V_{0}\right|+20 .
\end{aligned}
$$

Claim 3.2.2 We may assume that $\Delta(G)<n-1$.

Proof. Suppose on the contrary and let $w \in V(G)$ such that $\operatorname{deg}(w)=n-1$. Then by $\delta(G) \geq(n+1) / 2$ we have $\delta(G-w) \geq(n-1) / 2$, and thus $G-w$ has a hamiltonian cycle. This implies that $G$ has a spanning wheel subgraph, in particular, a spanning Halin subgraph of $G$. 
Claim 3.2.3 There exists a subgraph $T \subseteq G$ with $|V(T)| \equiv n(\bmod 2)$ such that $T$ and $G-V(T)$ satisfy the following conditions.

(i) $T$ is isomorphic to some graph in $\left\{T_{1}, T_{2}, \cdots, T_{5}\right\}$;

(ii) Let $2 m=n-|V(T)|$. Then $G-V(T)$ contains a balanced spanning bipartite graph $G^{\prime}$ with partite sets $U_{1}$ and $U_{2}$ such that $\left|U_{1}\right|=\left|U_{2}\right|=m$.

(iii) There exists a subset $W$ of $U_{1} \cup U_{2}$ with at most $\alpha_{2} n$ vertices such that $\operatorname{deg}_{G^{\prime}}\left(x, V\left(G^{\prime}\right)-\right.$ $W) \geq\left(1-\alpha_{1}-2 \alpha_{2}\right) m$ for all $x \notin W$.

(iv) Assume that $T$ has head link $x_{1} x_{2}$ and tail link $y_{1} y_{2}$. There exist $x_{1}^{\prime} x_{2}^{\prime}, y_{1}^{\prime} y_{2}^{\prime} \in E\left(G^{\prime}\right)$ such that $x_{i}^{\prime}, y_{i}^{\prime} \in U_{i}-W, x_{3-i}^{\prime} \sim x_{i}$, and $y_{3-i}^{\prime} \sim y_{i}$, for $i=1,2$; and if $T$ has a pendent vertex, then the pendent vertex is contained in $V_{1}^{\prime} \cup V_{2}^{\prime}-W$.

(v) There are $|W|$ vertex-disjoint 3-stars in $G^{\prime}-\left\{x_{1}^{\prime}, x_{2}^{\prime}, y_{1}^{\prime}, y_{2}^{\prime}\right\}$ with the vertices in $W$ as their centers.

Proof. By (1) and Lemma 3.3, for $i=1,2$, we notice that for any $u, v, w \in V_{i}^{\prime}$,

$$
\operatorname{deg}\left(u, v, w, V_{3-i}^{\prime}\right) \geq 3 \delta\left(V_{i}^{\prime}, V_{3-i}^{\prime}\right)-2\left|V_{3-i}^{\prime}\right| \geq\left(1 / 2-3 \alpha_{1}\right) n>n / 4 .
$$

We now separate the proof into two cases according to the parity of $n$.

Case 1. $n$ is even.

Suppose first that $\max \left\{\left|V_{1}^{\prime}\right|,\left|V_{2}^{\prime}\right|\right\} \leq n / 2$. We arbitrarily partition $V_{0}$ into $V_{10}$ and $V_{20}$ such that $\left|V_{1}^{\prime} \cup V_{10}\right|=\left|V_{2}^{\prime} \cup V_{20}\right|=n / 2$. Suppose $G\left[V_{1}^{\prime}\right]$ contains an edge $x_{1} u_{1}$ and there is a vertex $u_{2} \in \Gamma\left(u_{1}, V_{2}^{\prime}\right)$ such that $u_{2}$ is adjacent to a vertex $y_{2} \in V_{2}^{\prime}$. By (2), there exist distinct vertices $x_{2} \in \Gamma\left(x_{1}, u_{1}, V_{2}^{\prime}\right)-\left\{y_{2}, u_{1}\right\}, y_{1} \in \Gamma\left(y_{2}, u_{2}, V_{1}^{\prime}\right)-\left\{x_{1}, u_{1}\right\}$. Then $G\left[\left\{x_{1}, u_{1}, x_{2}, y_{1}, u_{1}, y_{2}\right\}\right]$ contains a subgraph $T$ isomorphic to $T_{1}$. So we assume $G\left[V_{1}^{\prime}\right]$ contains an edge $x_{1} u_{1}$ and no vertex in $\Gamma\left(u_{1}, V_{2}^{\prime}\right)$ is adjacent to any vertex in $V_{2}^{\prime}$. As $\delta(G) \geq(n+1) / 2, \delta\left(G\left[V_{2}^{\prime} \cup V_{20}\right]\right) \geq 1$. Let $u_{2} \in$ $\Gamma\left(u_{1}, V_{2}^{\prime}\right)$ and $u_{2} y_{2} \in E\left(G\left[V_{2}^{\prime} \cup V_{20}\right]\right)$. Since $\operatorname{deg}\left(u_{2}, V_{1}^{\prime}\right) \geq(n+1) / 2-\left|V_{0}\right|>\left|V_{1}^{\prime} \cup V_{10}\right|-\left|V_{0}\right|$ and $\operatorname{deg}\left(y_{2}, V_{1}^{\prime}\right) \geq 3\left|V_{0}\right|+10, \operatorname{deg}\left(u_{2}, y_{2}, V_{1}^{\prime} \cup V_{10}\right) \geq 2\left|V_{0}\right|+10$. Let $x_{2} \in \Gamma\left(x_{1}, u_{1}, V_{2}^{\prime}\right)-\left\{y_{2}, u_{2}\right\}, y_{1} \in$ $\Gamma\left(y_{2}, u_{2}, V_{1}^{\prime}\right)-\left\{x_{1}, u_{1}\right\}$. Then $G\left[\left\{x_{1}, u_{1}, x_{2}, y_{1}, u_{2}, y_{2}\right\}\right]$ contains a subgraph $T$ isomorphic to $T_{1}$. By symmetry, we can find $T \cong T_{1}$ if $G\left[V_{2}^{\prime}\right]$ contains an edge. Hence we assume that both $V_{1}^{\prime}$ and $V_{2}^{\prime}$ are independent sets. Again, as $\delta(G) \geq(n+1) / 2, \delta\left(G\left[V_{1}^{\prime} \cup V_{10}\right]\right), \delta\left(G\left[V_{2}^{\prime} \cup V_{20}\right]\right) \geq 1$. Let $x_{1} u_{1} \in E\left(G\left[V_{1}^{\prime} \cup V_{10}\right]\right)$ and $y_{2} u_{2} \in E\left(G\left[V_{2}^{\prime} \cup V_{20}\right]\right)$ such that $x_{1} \in V_{1}^{\prime}$ and $u_{2} \in \Gamma\left(u_{1}, V_{2}^{\prime}\right)$. Since $\operatorname{deg}\left(x_{1}, V_{2}^{\prime}\right) \geq(n+1) / 2-\left|V_{0}\right|>\left|V_{2}^{\prime} \cup V_{20}\right|-\left|V_{0}\right|$ and $\operatorname{deg}\left(u_{1}, V_{2}^{\prime}\right) \geq 3\left|V_{0}\right|+10$, we have $\operatorname{deg}\left(x_{1}, u_{1}, V_{2}^{\prime}\right) \geq 2\left|V_{0}\right|+10$. Hence, there exists $x_{2} \in \Gamma\left(x_{1}, u_{1}, V_{2}^{\prime}\right)-\left\{y_{2}, u_{2}\right\}$. Similarly, there exists $y_{1} \in \Gamma\left(y_{2}, u_{2}, V_{1}^{\prime}\right)-\left\{x_{1}, u_{1}\right\}$. Then $G\left[\left\{x_{1}, u_{1}, x_{2}, y_{1}, u_{2}, y_{2}\right\}\right]$ contains a subgraph $T$ isomorphic to $T_{1}$. Let $m=(n-6) / 2, U_{1}=\left(V_{1}^{\prime} \cup V_{10}\right)-V(T)$ and $U_{2}=\left(V_{2}^{\prime} \cup V_{20}\right)-V(T)$, and $W=V_{0}-V(T)$. We then have $\left|U_{1}\right|=\left|U_{2}\right|=m$.

Let $G^{\prime}=\left(V(G)-V(T), E_{G}\left(U_{1}, U_{2}\right)\right)$ be the bipartite graph with partite sets $U_{1}$ and $U_{2}$. Notice that $|W| \leq\left|V_{0}\right| \leq \alpha_{2}\left|V_{2}\right|<\alpha_{2} n$. By (1), we have $\operatorname{deg}_{G^{\prime}}\left(x, V\left(G^{\prime}\right)-W\right) \geq\left(1-\alpha_{1}-\right.$ $\left.2 \alpha_{2}\right) m$ for all $x \notin W$. This shows (iii). By the construction of $T$ above, we have $x_{1}, y_{1} \in$ $V_{1}^{\prime}-W$. Let $i=1,2$. By (1), we have $\delta\left(V_{0}, U_{i}-W\right)=\delta\left(V_{0}, V_{i}^{\prime}-V(T)\right) \geq 3\left|V_{0}\right|+6$. So $\left|\Gamma_{G^{\prime}}\left(y_{2}, U_{1}-W\right)\right|,\left|\Gamma_{G^{\prime}}\left(x_{2}, U_{1}-W\right)\right| \geq 3\left|V_{0}\right|+6$. Applying statement (iii) and Lemma 3.3, we have $e_{G^{\prime}}\left(\Gamma_{G^{\prime}}\left(x_{2}, U_{1}-W\right), \Gamma_{G^{\prime}}\left(x_{1}, U_{2}-W\right)\right), e_{G^{\prime}}\left(\Gamma_{G^{\prime}}\left(y_{2}, U_{1}-W\right), \Gamma_{G^{\prime}}\left(y_{1}, U_{2}-W\right)\right) \geq\left(3\left|V_{0}\right|+\right.$ $6)\left(1-2 \alpha_{1}-4 \alpha_{2}\right) m>2 m$. Hence, we can find independent edges $x_{1}^{\prime} x_{2}^{\prime}$ and $y_{1}^{\prime} y_{2}^{\prime}$ such that $x_{i}^{\prime}, y_{i}^{\prime} \in U_{i}-W, x_{3-i}^{\prime} \sim x_{i}$, and $y_{3-i}^{\prime} \sim y_{i}$. This gives statement (iv). Finally, as $\delta\left(V_{0}, U_{i}-W\right) \geq$ $3\left|V_{0}\right|+6$, we have $\delta\left(V_{0}, U_{i}-W-\left\{x_{1}^{\prime}, x_{2}^{\prime}, y_{1}^{\prime}, y_{2}^{\prime}\right\}\right) \geq 3\left|V_{0}\right|+2$. Hence, there are vertex-disjoint 3 -stars with their centers in $W$. 
Otherwise we have $\max \left\{\left|V_{1}^{\prime}\right|,\left|V_{2}^{\prime}\right|\right\}>n / 2$. By (1), we have the same lower bound for $\delta\left(V_{1}^{\prime}, V_{2}^{\prime}\right), \delta\left(V_{2}^{\prime}, V_{1}^{\prime}\right)$, and $\delta\left(V_{0}, V_{1}^{\prime}\right), \delta\left(V_{0}, V_{2}^{\prime}\right)$. Furthermore, all the argument in the following will depend only on the degree conditions, so we assume, w.l.o.g., that $\left|V_{1}^{\prime}\right| \geq n / 2+1$. Then $\delta\left(G\left[V_{1}^{\prime}\right]\right) \geq 2$ and thus $G\left[V_{1}^{\prime}\right]$ contains two vertex-disjoint paths isomorphic to $P_{3}$ and $P_{2}$, respectively. Let $m=(n-8) / 2$. We consider three cases here. Case (a): $\left|V_{1}^{\prime}\right|-5 \leq m$. Then let $x_{1} u_{1} w_{1}, y_{1} v_{1} \subseteq G\left[V_{1}^{\prime}\right]$ be two vertex-disjoint paths, and let $x_{2} \in \Gamma\left(x_{1}, u_{1}, w_{1}, V_{2}^{\prime}\right), y_{2} \in$ $\Gamma\left(y_{1}, v_{1}, V_{2}^{\prime}\right)$ and $z \in \Gamma\left(w_{1}, v_{1}, V_{2}^{\prime}\right)$ be three distinct vertices. Then $G\left[\left\{x_{1}, u_{1}, w_{1}, x_{2}, z, y_{1}, v_{1}, y_{2}\right\}\right]$ contains a subgraph $T$ isomorphic to $T_{4}$. Notice that $\left|V_{2}^{\prime}-V(T)\right| \leq m$. We arbitrarily partition $V_{0}$ into $V_{10}$ and $V_{20}$ such that $\left|V_{1}^{\prime} \cup V_{10}\right|=\left|V_{2}^{\prime} \cup V_{20}\right|=m$. Let $U_{1}=\left(V_{1}^{\prime} \cup V_{10}\right)-V(T)$, $U_{2}=\left(V_{2}^{\prime} \cup V_{20}\right)-V(T)$, and $W=V_{0}$. Hence we assume $\left|V_{1}^{\prime}\right|-5=m+t_{1}$ for some $t_{1} \geq 1$. This implies that $\left|V_{1}^{\prime}\right|=n / 2+t_{1}+1$ and thus $\delta\left(G\left[V_{1}^{\prime}\right]\right) \geq t_{1}+2$. Let $V_{1}^{0}$ be the set of vertices $u \in V_{1}^{\prime}$ such that $\operatorname{deg}\left(u, V_{1}^{\prime}\right) \geq \alpha_{1} m$. Case $(\mathrm{b}):\left|V_{1}^{0}\right| \geq\left|V_{1}^{\prime}\right|-5-m$. Then we form a set $W$ with $\left|V_{1}^{\prime}\right|-5-m$ vertices from $V_{1}^{0}$ and all the vertices of $V_{0}$. Then $\left|V_{1}^{\prime}-W\right|=m+5+t_{1}-\left(\left|V_{1}^{\prime}\right|-5-m\right)=m+5=n / 2+1$, and hence $\delta\left(G\left[V_{1}^{\prime}-W\right]\right) \geq 2$. Similarly as in Case (a), we can find a subgraph $T$ of $G$ contained in $G\left[\left(V_{1}^{\prime} \cup V_{2}^{\prime}\right)-W\right]$ isomorphic to $T_{4}$. Let $U_{1}=V_{1}^{\prime}-V(T)-W, U_{2}=\left(V_{2}^{\prime} \cup W\right)-V(T)$. Then $\left|U_{1}\right|=\left|U_{2}\right|=m$. Thus we have Case (c): $\left|V_{1}^{0}\right|<\left|V_{1}^{\prime}\right|-5-m$. Suppose that $\left|V_{1}^{\prime}-V_{1}^{0}\right|=m+5+t_{1}^{\prime}=n / 2+t_{1}^{\prime}+1$ for some $t_{1}^{\prime} \geq 1$. This implies that $\delta\left(G\left[V_{1}^{\prime}-V_{1}^{0}\right]\right) \geq t_{1}^{\prime}+2$.

We show that $G\left[V_{1}^{\prime}-V_{1}^{0}\right]$ contains $t_{1}^{\prime}+2$ vertex-disjoint 3 -stars. To see this, suppose $G\left[V_{1}^{\prime}-V_{1}^{0}\right]$ contains a subgraph $M$ of at most $s<t_{1}^{\prime}+23$-stars. By counting the number of edges between $V(M)$ and $V_{1}^{\prime}-V_{1}^{0}-V(M)$ in two ways, we get that $t_{1}^{\prime}\left|V_{1}^{\prime}-V_{1}^{0}-V(M)\right| \leq$ $e_{G-V_{1}^{0}}\left(V(M), V_{1}^{\prime}-V_{1}^{0}-V(M)\right) \leq 4 s \Delta\left(G\left[V_{1}^{\prime}-V_{1}^{0}\right]\right) \leq 4 s \alpha_{1} m$. Since $\left|V_{1}^{\prime}-V_{1}^{0}\right|=m+5+t_{1}^{\prime}=$ $n / 2+t_{1}^{\prime}+1,\left|V_{1}^{\prime}-V_{1}^{0}-V(M)\right| \geq m-3 t_{1}^{\prime} \geq m-6 \alpha_{2} m$, where the last inequality holds as $\left|V_{1}^{\prime}\right| \leq(1 / 2+\beta) n+\alpha_{2}\left|V_{2}^{\prime}\right|$ implying that $t_{1}^{\prime} \leq\left|V_{1}^{\prime}\right|-m-5 \leq 2 \alpha_{2} m$. This, together with the assumption that $\alpha \leq(1 / 8)^{3}$ gives that $s \geq t_{1}^{\prime}+2$, showing a contradiction. Hence we have $s \geq t_{1}^{\prime}+2$. Let $x_{1} u_{1} w_{1}$ and $y_{1} v_{1}$ be two paths taken from two 3 -stars in $M$. Then we can find a subgraph $T$ of $G$ isomorphic to $T_{4}$ in the same way as in Case (a). We take exactly $t_{1}^{\prime} 3$-stars from the remaining ones in $M$ and denote the centers of these stars by $W^{\prime}$. Let $U_{1}=V_{1}^{\prime}-V_{1}^{0}-V(T)-W^{\prime}, W=W^{\prime} \cup V_{1}^{0} \cup V_{0}$, and $U_{2}=\left(V_{2}^{\prime} \cup W\right)-V(T)$. Then $\left|U_{1}\right|=\left|U_{2}\right|=m$.

For the partition of $U_{1}$ and $U_{2}$ in all the cases discussed in the paragraph above, we let $G^{\prime}=\left(V(G)-V(T), E_{G}\left(U_{1}, U_{2}\right)\right)$ be the bipartite graph with partite sets $U_{1}$ and $U_{2}$. Notice that $|W| \leq\left|V_{0}\right| \leq \alpha_{2} n$ if Case (a) occurs, $|W| \leq\left|V_{0}\right|+\left|V_{1}^{\prime}\right|-m-5 \leq(1 / 2+\beta) n+\mid V_{0} \cup$ $V_{01} \mid-n / 2-1 \leq \alpha_{2} n$ if Case (b) occurs, and $|W|=\left|W^{\prime} \cup V_{1}^{0} \cup V_{0}\right|=\left|V_{1}^{\prime}-U_{1}-V(T)\right|+$ $\left|V_{0} \cup V_{01}\right| \leq(1 / 2+\beta) n-(1 / 2-4) n+\left|V_{0} \cup V_{01}\right| \leq \alpha_{2} n$ if Case (c) occurs. (Recall that $\left|V_{1}^{\prime}\right| \leq(1 / 2+\beta) n+\left|V_{01}\right|$ and $\left|V_{0} \cup V_{01}\right| \leq \alpha_{2}\left|V_{2}\right|$ from (1).) Since $\delta\left(V_{2}^{\prime}, V_{1}^{\prime}\right) \geq\left(1-2 \alpha_{1} / 3\right) n$ from (1) and $\left|V_{1}^{\prime}-U_{1}\right| \leq 2 \alpha_{2} m$, we have $\delta\left(U_{2}-W, U_{1}-W\right) \geq\left(1-\alpha_{1}-2 \alpha_{2}\right) m$. On the other hand, from (1), $\delta\left(V_{1}^{\prime}, V_{2}^{\prime}\right) \geq\left(1 / 2-2 \alpha_{1} / 3\right) n$. This gives that $\delta\left(U_{1}-W, U_{2}-W\right) \geq\left(1-\alpha_{1}-2 \alpha_{2}\right) m$. Hence, we have $\operatorname{deg}_{G^{\prime}}\left(x, V\left(G^{\prime}\right)-W\right) \geq\left(1-\alpha_{1}-2 \alpha_{2}\right) m$ for all $x \notin W$. According to the construction of $T$, we have $x_{1}, y_{1} \in V_{1}^{\prime}-W$. Applying statement (iii) and Lemma 3.3, we have $e_{G^{\prime}}\left(\Gamma_{G^{\prime}}\left(x_{1}, U_{2}-W\right), \Gamma_{G^{\prime}}\left(x_{2}, U_{1}-W\right)\right), e_{G^{\prime}}\left(\Gamma_{G^{\prime}}\left(y_{1}, U_{2}-W\right), \Gamma_{G^{\prime}}\left(y_{2}, U_{1}-W\right)\right) \geq\left(3\left|V_{0}\right|+\right.$ $6)\left(1-2 \alpha_{1}-4 \alpha_{2}\right) m>2 m$. Hence, we can find independent edges $x_{1}^{\prime} x_{2}^{\prime}$ and $y_{1}^{\prime} y_{2}^{\prime}$ such that $x_{i}^{\prime}, y_{i}^{\prime} \in U_{i}-W, x_{3-i}^{\prime} \sim x_{i}$, and $y_{3-i}^{\prime} \sim y_{i}$. By the construction of $T, T$ is isomorphic to $T_{4}$, and the pendent vertex $z \in V_{2}^{\prime} \subseteq V_{1}^{\prime} \cup V_{2}^{\prime}-W$. This gives statement (iv). Finally, as

$$
\begin{aligned}
\delta\left(V_{0}, U_{1}-W\right) & \geq \delta\left(V_{0}, V_{1}^{\prime}\right)-\left|V_{1}^{\prime}-\left(U_{1}-W\right)\right| \geq \alpha_{1} n / 3-\left(1 / 2+\alpha_{2}\right) n+n / 2-4-\alpha_{2} n \\
& \geq\left(1 / 3 \alpha_{1}-2 \alpha_{2}\right) n-4 \geq 3|W|+5,
\end{aligned}
$$


we have $\delta\left(V_{0}, U_{1}-W-\left\{x_{1}^{\prime}, x_{2}^{\prime}, y_{1}^{\prime}, y_{2}^{\prime}\right\}\right) \geq 3|W|+1$. By the definition of $V_{1}^{0}$, we have $\delta\left(V_{1}^{0}, V_{1}^{\prime}-\right.$ $\left.W-\left\{x_{1}^{\prime}, x_{2}^{\prime}, y_{1}^{\prime}, y_{2}^{\prime}\right\}\right) \geq \alpha_{1} m-\alpha_{2} n-4 \geq 3|W|$. For the vertices in $W^{\prime}$ in Case (c), we already know that there are vertex-disjoint 3 -stars in $G^{\prime}$ with centers in $W^{\prime}$. Hence, regardless of the construction of $W$, we can always find vertex-disjoint 3 -stars with their centers in $W$.

Case 2. $n$ is odd.

Suppose first that $\max \left\{\left|V_{1}^{\prime}\right|,\left|V_{2}^{\prime}\right|\right\} \leq(n+1) / 2$ and let $m=(n-7) / 2$. We arbitrarily partition $V_{0}$ into $V_{10}$ and $V_{20}$ such that, w.l.o.g., say $\left|V_{1}^{\prime} \cup V_{10}\right|=(n+1) / 2$ and $\left|V_{2}^{\prime} \cup V_{20}\right|=$ $(n-1) / 2$. (Again, here we use the symmetry of the lower bounds on $\delta\left(V_{1}^{\prime}, V_{2}^{\prime}\right), \delta\left(V_{2}^{\prime}, V_{1}^{\prime}\right)$, and $\delta\left(V_{0}, V_{1}^{\prime}\right), \delta\left(V_{0}, V_{2}^{\prime}\right)$ from (1).) We show that $G\left[V_{1}^{\prime} \cup V_{10}\right]$ either contains two independent edges or is isomorphic to $K_{1,(n-1) / 2}$. As $\delta(G) \geq(n+1) / 2$, we have $\delta\left(G\left[V_{1}^{\prime} \cup V_{10}\right]\right) \geq 1$. Since $n$ is sufficiently large, $(n+1) / 2>3$. Then it is easy to see that if $G\left[V_{1}^{\prime} \cup V_{10}\right] \neq$ $K_{1,(n-1) / 2}$, then $G\left[V_{1}^{\prime} \cup V_{10}\right]$ contains two independent edges. Furthermore, we can choose two independent edges $x_{1} u_{1}$ and $y_{1} v_{1}$ such that $u_{1}, v_{1} \in V_{1}^{\prime}$. This is obvious if $\left|V_{10}\right| \leq 1$. So we assume $\left|V_{10}\right| \geq 2$. As $\delta\left(V_{0}, V_{1}^{\prime}\right) \geq 3\left|V_{0}\right|+10$, by choosing $x_{1}, y_{1} \in V_{10}$, we can choose distinct vertices $u_{1} \in \Gamma\left(x_{1}, V_{1}^{\prime}\right)$ and $v_{1} \in \Gamma\left(y_{1}, V_{1}^{\prime}\right)$. Let $x_{2} \in \Gamma\left(x_{1}, u_{1}, V_{2}^{\prime}\right), y_{2} \in \Gamma\left(y_{1}, v_{1}, V_{2}^{\prime}\right)$ and $z \in \Gamma\left(u_{1}, v_{1}, V_{2}^{\prime}\right)$. Then $G\left[\left\{x_{1}, u_{1}, x_{2}, y_{1}, v_{1}, y_{2}, z\right\}\right]$ contains a subgraph $T$ isomorphic to $T_{3}$. We assume now that $G\left[V_{1}^{\prime} \cup V_{10}\right]$ is isomorphic to $K_{1,(n-1) / 2}$. Let $u_{1}$ be the center of the star $K_{1,(n-1) / 2}$. Then each leaf of the star has at least $(n-1) / 2$ neighbors in $V_{2}^{\prime} \cup V_{20}$. Since $\left|V_{2}^{\prime} \cup V_{20}\right|=(n-1) / 2$, we have $\Gamma\left(v, V_{2}^{\prime} \cup V_{20}\right)=V_{2}^{\prime} \cup V_{20}$ if $v \in V_{1}^{\prime} \cup V_{10}-\left\{u_{1}\right\}$. By the definition of $V_{0}, \Delta\left(V_{0}, V_{1}^{\prime}\right)<\left(1-\alpha_{1}\right)\left|V_{1}\right|+\left|V_{01}\right|$ and $\Delta\left(V_{0}, V_{2}^{\prime}\right)<\left(1-\alpha_{1}\right)\left|V_{2}^{\prime}\right|$, and so $u_{1} \in V_{1}^{\prime}, V_{10}=\emptyset$ and $V_{20}=\emptyset$. We claim that $V_{2}^{\prime}$ is not an independent set. Otherwise, by $\delta(G) \geq(n+1) / 2$, for each $v \in V_{2}^{\prime}, \Gamma\left(v, V_{1}^{\prime}\right)=V_{1}^{\prime}$. This in turn shows that $u_{1}$ has degree $n-1$, showing a contradiction to Claim 3.2.2. So let $y_{2} v_{2} \in E\left(G\left[V_{2}^{\prime}\right]\right)$ be an edge. Let $w_{1} \in$ $\Gamma\left(v_{2}, V_{1}^{\prime}\right)-\left\{u_{1}\right\}$ and $w_{1} u_{1} x_{1}$ be the path containing $w_{1}$. Choose $y_{1} \in \Gamma\left(y_{2}, v_{2}, V_{1}^{\prime}\right)-\left\{w_{1}, u_{1}, x_{1}\right\}$ and $x_{2} \in \Gamma\left(x_{1}, u_{1}, w_{1}, V_{2}^{\prime}\right)-\left\{y_{2}, v_{2}\right\}$. Then $G\left[\left\{x_{1}, u_{1}, x_{2}, w_{1}, v_{2}, y_{2}, y_{1}\right\}\right]$ contains a subgraph $T$ isomorphic to $T_{2}$. Let $U_{1}=\left(V_{1}^{\prime} \cup V_{10}\right)-V(T)$ and $U_{2}=\left(V_{2}^{\prime} \cup V_{20}\right)-V(T)$ and $W=V_{0}-V(T)$. We have $\left|U_{1}\right|=\left|U_{2}\right|=m$ and $|W| \leq\left|V_{0}\right| \leq \alpha_{2} n$.

Otherwise we have $\max \left\{\left|V_{1}^{\prime}\right|,\left|V_{2}^{\prime}\right|\right\} \geq(n+1) / 2+1$. By the symmetry of lower bounds on degrees related to $V_{1}^{\prime}$ and $V_{2}^{\prime}$ from (1), we assume, w.l.o.g., that $\left|V_{1}^{\prime}\right| \geq(n+1) / 2+1$. Then $\delta\left(G\left[V_{1}^{\prime}\right]\right) \geq 2$ and thus $G\left[V_{1}^{\prime}\right]$ contains two independent edges. Let $m=(n-7) / 2$ and $V_{1}^{0}$ be the set of vertices $u \in V_{1}^{\prime}$ such that $\operatorname{deg}\left(u, V_{1}^{\prime}\right) \geq \alpha_{1} m$. Since $\left|V_{1}^{\prime}\right| \geq(n+1) / 2+1>m+4$, we assume $\left|V_{1}^{\prime}\right|=m+4+t_{1}$ for some $t_{1} \geq 1$. We consider three cases here.

Case (a): $\left|V_{1}^{0}\right| \geq\left|V_{1}^{\prime}\right|-m-4$. We form a set $W$ with $\left|V_{1}^{\prime}\right|-4-m$ vertices from $V_{1}^{0}$ and all the vertices of $V_{0}$. Then $\left|V_{1}^{\prime}-W\right|=m+4+t_{1}-\left(\left|V_{1}^{\prime}\right|-4-m\right)=m+4=(n+1) / 2$, and we have $\delta\left(G\left[V_{1}^{\prime}-W\right]\right) \geq 1$. As any vertex $u \in V_{1}^{\prime}-W$ is a vertex such that $\operatorname{deg}\left(u, V_{1}^{\prime}\right)<\alpha_{1} m$, we know $G\left[V_{1}^{\prime}-W\right]$ contains two independent edges. Let $x_{1} u_{1}, y_{1} v_{1} \subseteq E\left(G\left[V_{1}^{\prime}-W\right]\right)$ be two independent edges, and let $x_{2} \in \Gamma\left(x_{1}, u_{1}, V_{2}^{\prime}\right), y_{2} \in \Gamma\left(y_{1}, v_{1}, V_{2}^{\prime}\right)$ and $z \in \Gamma\left(w_{1}, v_{1}, V_{2}^{\prime}\right)$ be three distinct vertices. Then $G\left[\left\{x_{1}, u_{1}, x_{2}, z, y_{1}, v_{1}, y_{2}\right\}\right]$ contains a subgraph $T$ isomorphic to $T_{3}$. Let $U_{1}=V_{1}^{\prime}-V(T)-W, U_{2}=\left(V_{2}^{\prime} \cup W\right)-V(T)$. Then $\left|U_{1}\right|=\left|U_{2}\right|=m$ and $|W| \leq\left|V_{0}\right|+\left|V_{1}^{\prime}-U_{1}\right| \leq\left|V_{2}-V_{2}^{\prime}\right|+\beta n+4 \leq \alpha_{2} n$.

Thus we have $\left|V_{1}^{0}\right|<\left|V_{1}^{\prime}\right|-4-m$. Suppose that $\left|V_{1}^{\prime}-V_{1}^{0}\right|=m+4+t_{1}^{\prime}=(n+1) / 2+t_{1}^{\prime}$ for some $t_{1}^{\prime} \geq 1$. This implies that $\delta\left(G\left[V_{1}^{\prime}-V_{1}^{0}\right]\right) \geq t_{1}^{\prime}+1$.

Case (b): $t_{1}^{\prime} \geq 2$. We show that $G\left[V_{1}^{\prime}-V_{1}^{0}\right]$ contains $t_{1}^{\prime}+2$ vertex-disjoint 3 -stars. To see this, suppose $G\left[V_{1}^{\prime}-V_{1}^{0}\right]$ contains a subgraph $M$ of at most $s$ vertex disjoint 3 -stars. We may assume that $s<t_{1}^{\prime}+2$. Then we have $\left(t_{1}-1\right)\left|V_{1}^{\prime}-V_{1}^{0}-V(M)\right| \leq e_{G-V_{1}^{0}}\left(V(M), V_{1}^{\prime}-V_{1}^{0}-\right.$ $V(M)) \leq 4 s \Delta\left(G\left[V_{1}^{\prime}-V_{1}^{0}\right]\right)$. Since $\left|V_{1}^{\prime}-V_{1}^{0}\right|=m+4+t_{1}^{\prime}=(n+1) / 2+t_{1}^{\prime},\left|V_{1}^{\prime}-V_{1}^{0}-V(M)\right| \geq$ $m-3 t_{1}^{\prime} \geq m-6 \alpha_{2} m$, where the last inequality holds as $\left|V_{1}^{\prime}\right| \leq(1 / 2+\beta) n+\alpha_{2}\left|V_{2}^{\prime}\right|$ implying 
that $t_{1}^{\prime} \leq\left|V_{1}^{\prime}\right|-m-4 \leq 2 \alpha_{2} m$. This, together with the assumption that $\alpha \leq(1 / 8)^{3}$ gives that $s \geq t_{1}^{\prime}+2$, showing a contradiction. Hence we have $s \geq t_{1}^{\prime}+2$. Let $x_{1} u_{1}$ and $y_{1} v_{1}$ be two paths taken from two 3 -stars in $M$. Then we can find a subgraph $T$ of $G$ isomorphic to $T_{3}$ the same way as in Case (a). We take exactly $t_{1}^{\prime} 3$-stars from the remaining ones in $M$ and denote the centers of these stars by $W^{\prime}$. Let $U_{1}=V_{1}^{\prime}-V_{1}^{0}-V(T)-W^{\prime}, W=W^{\prime} \cup V_{1}^{0} \cup V_{0}$, and $U_{2}=\left(V_{2}^{\prime} \cup W\right)-V(T)$. Then $\left|U_{1}\right|=\left|U_{2}\right|=m$.

Case (c): $t_{1}^{\prime}=1$. In this case, we let $m=(n-9) / 2$. If $G\left[V_{1}^{\prime}-V_{1}^{0}\right]$ contains a vertex adjacent to all other vertices in $V_{1}^{\prime}-V_{1}^{0}$, then the vertex would be contained in $V_{1}^{0}$ by the definition of $V_{1}^{0}$. Hence, we assume that $G\left[V_{1}^{\prime}-V_{1}^{0}\right]$ has no vertex adjacent to all other vertices in $V_{1}^{\prime}-V_{1}^{0}$. Then by the assumptions that $\delta(G) \geq(n+1) / 2$ and $\left|V_{1}^{\prime}-V_{1}^{0}\right|=(n+1) / 2+1$, we can find two copies of vertex disjoint $P_{3} \mathrm{~s}$ in $G\left[V_{1}^{\prime}-V_{1}^{0}\right]$. Let $x_{1} u_{1} w_{1}$ and $y_{1} v_{1} z_{1}$ be two $P_{3}$ s in $G\left[V_{1}^{\prime}-V_{1}^{0}\right]$. There exist distinct vertices $x_{2} \in \Gamma\left(x_{1}, u_{1}, w_{1}, V_{2}^{\prime}\right), y_{2} \in \Gamma\left(y_{1}, v_{1}, z_{1}, V_{2}^{\prime}\right)$ and $z \in \Gamma\left(w_{1}, z_{1}, V_{2}^{\prime}\right)$. Then $G\left[\left\{x_{1}, u_{1}, w_{1}, x_{2}, y_{1}, v_{1}, z_{1}, y_{2}, z\right\}\right]$ contains a subgraph $T$ isomorphic to $T_{5}$. Let $U_{1}=V_{1}^{\prime}-V_{1}^{0}-V(T), W=V_{1}^{0} \cup V_{0}$, and $U_{2}=\left(V_{2}^{\prime} \cup W\right)-V(T)$. Then $\left|U_{1}\right|=\left|U_{2}\right|=m$.

For the partition of $U_{1}$ and $U_{2}$ in all the cases discussed in Case 2, we let $G^{\prime}=(V(G)-$ $\left.V(T), E_{G}\left(U_{1}, U_{2}\right)\right)$ be the bipartite graph with partite sets $U_{1}$ and $U_{2}$. Similarly as in Case 1, we can show that all the statements (i)-(v) hold.

Let $W_{1}=U_{1} \cap W$ and $W_{2}=U_{2} \cap W$. By (v) of Claim 3.2.3, we know that there are $\left|W_{1}\right|$ vertex-disjoint 3-stars with centers in $W_{1}$ and all other vertices in $U_{2}-W_{2}-\left\{x_{1}^{\prime}, y_{1}^{\prime}, x_{2}^{\prime}, y_{2}^{\prime}\right\}$, and $\left|W_{2}\right|$ vertex-disjoint 3 -stars with centers in $W_{2}$ and all other vertices in $U_{1}-W_{1}-\left\{x_{1}^{\prime}, y_{1}^{\prime}, x_{2}^{\prime}, y_{2}^{\prime}\right\}$, and all these $\left|W_{1}\right|+\left|W_{2}\right|$ stars are vertex-disjoint. Let $S$ be the union of the 3 -stars with centers in $W_{2}$.

For any $u, v \in U_{2}-W_{2}, \Gamma\left(u, v, U_{1}-W_{1}-V(S)-\left\{x_{1}^{\prime}, x_{2}^{\prime}, y_{1}^{\prime}, y_{2}^{\prime}\right\}\right) \geq 3\left|W_{1}\right|$, and for any $u, v, w \in U_{1}-W_{1}-V(S), \Gamma\left(u, v, w, U_{2}-V(S)-\left\{x_{1}^{\prime}, x_{2}^{\prime}, y_{1}^{\prime}, y_{2}^{\prime}\right\}\right) \geq 4\left|W_{1}\right|$. By Lemma 3.2, we can find a ladder $L_{1}$ disjoint from the 3 -stars in $S$ with centers in $W_{2}$ such that $L_{1}$ is spanning on $W_{1}, 4\left|W_{1}\right|-1$ vertices from $U_{2}-W_{2}-\left\{x_{1}^{\prime}, x_{2}^{\prime}, y_{1}^{\prime}, y_{2}^{\prime}\right\}$, and another $3\left|W_{1}\right|-1$ vertices from $U_{1}-W_{1}-\left\{x_{1}^{\prime}, x_{2}^{\prime}, y_{1}^{\prime}, y_{2}^{\prime}\right\}$, if $W_{1} \neq \emptyset$.

For any $u, v \in U_{1}-W_{1}, \Gamma\left(u, v, U_{2}-W_{2}-V\left(L_{1}\right)-\left\{x_{1}^{\prime}, x_{2}^{\prime}, y_{1}^{\prime}, y_{2}^{\prime}\right\}\right) \geq 3\left|W_{2}\right|$, and for any $u, v, w \in U_{2}-W_{2}, \Gamma\left(u, v, w, U_{1}-W_{1}-V\left(L_{1}\right)-\left\{x_{1}^{\prime}, x_{2}^{\prime}, y_{1}^{\prime}, y_{2}^{\prime}\right\}\right) \geq 4\left|W_{2}\right|$. By Lemma 3.2, we can find a ladder $L_{2}$ disjoint from $L_{1}$ such that $L_{2}$ is spanning on $W_{2}, 4\left|W_{2}\right|-1$ vertices from $U_{1}-V\left(L_{1}\right)-\left\{x_{1}^{\prime}, x_{2}^{\prime}, y_{1}^{\prime}, y_{2}^{\prime}\right\}$, and another $3\left|W_{2}\right|-1$ vertices from $U_{2}-V\left(L_{1}\right)-\left\{x_{1}^{\prime}, x_{2}^{\prime}, y_{1}^{\prime}, y_{2}^{\prime}\right\}$, if $W_{2} \neq \emptyset$.

Denote $a_{1 i} a_{2 i}$ and $b_{1 i} b_{2 i}$ the first and last rungs of $L_{i}$ (if $L_{i}$ exists), respectively, where $a_{1 i}, b_{1 i} \in U_{1}$. As $\left|U_{1}\right|=\left|U_{2}\right|$, and we took $4\left|W_{1}\right|+4\left|W_{2}\right|-2$ vertices respectively from $U_{1}$ and $U_{2}$ when constructing $L_{1}$ and $L_{2}$, we have $\left|U_{1}-V\left(L_{1} \cup L_{2}\right)\right|=\left|U_{2}-V\left(L_{1} \cup L_{2}\right)\right|$. Let

$$
U_{i}^{\prime}=U_{i}-V\left(L_{1} \cup L_{2}\right), \quad m^{\prime}=\left|U_{1}^{\prime}\right|=\left|U_{2}^{\prime}\right|, \quad \text { and } \quad G^{\prime \prime}=G^{\prime \prime}\left(U_{1}^{\prime} \cup U_{2}^{\prime}, E_{G}\left(U_{1}^{\prime}, U_{2}^{\prime}\right)\right) .
$$

Since $|W| \leq \alpha_{2} n, m \geq(n-9) / 2$, and $n$ is sufficiently large, we have $1 / n+7|W| \leq 15 \alpha_{2} m$. As $\delta\left(G^{\prime}-W\right) \geq\left(1-\alpha_{1}-2 \alpha_{2}\right) m$ and $\alpha \leq(1 / 17)^{3}$, we obtain the following:

$$
\delta\left(G^{\prime \prime}\right) \geq 7 m^{\prime} / 8+1 .
$$

Let $a_{2 i}^{\prime} \in \Gamma\left(a_{1 i}, U_{2}^{\prime}\right), a_{1 i}^{\prime} \in \Gamma\left(a_{2 i}, U_{1}^{\prime}\right)$ such that $a_{1 i}^{\prime} a_{2 i}^{\prime} \in E(G) ;$ and $b_{2 i}^{\prime} \in \Gamma\left(b_{1 i}, U_{2}^{\prime}\right), b_{1 i}^{\prime} \in$ $\Gamma\left(b_{2 i}, U_{1}^{\prime}\right)$ such that $b_{1 i}^{\prime} b_{2 i}^{\prime} \in E(G)$. We have the claim below.

Claim 3.2.4 The balanced bipartite graph $G^{\prime \prime}$ contains three vertex-disjoint ladders $Q_{1}, Q_{2}$, and $Q_{3}$ spanning on $V\left(G^{\prime \prime}\right)$ such that the first rung of $Q_{1}$ is $x_{1}^{\prime} x_{2}^{\prime}$ and the last rung of $Q_{1}$ is 
$a_{11}^{\prime} a_{21}^{\prime}$, the first rung of $Q_{2}$ is $b_{11}^{\prime} b_{21}^{\prime}$ and the last rung of $Q_{2}$ is $a_{12}^{\prime} a_{22}^{\prime}$, the first rung of $Q_{3}$ is $b_{12}^{\prime} b_{22}^{\prime}$ and the last rung of $Q_{3}$ is $y_{1}^{\prime} y_{2}^{\prime}$.

Proof. Since $\delta\left(G^{\prime \prime}\right) \geq 7 m^{\prime} / 8+1>m^{\prime} / 2+6, G^{\prime \prime}$ has a perfect matching $M$ containing the following edges: $x_{1}^{\prime} x_{2}^{\prime}, a_{11}^{\prime} a_{21}^{\prime}, b_{11}^{\prime} b_{21}^{\prime}, a_{12}^{\prime} a_{22}^{\prime}, b_{12}^{\prime} b_{22}^{\prime}, y_{1}^{\prime} y_{2}^{\prime}$. We identify $a_{11}^{\prime}$ and $b_{11}^{\prime}, a_{21}^{\prime}$ and $b_{21}^{\prime}, a_{12}^{\prime}$ and $b_{12}^{\prime}$, and $a_{22}^{\prime}$ and $b_{22}^{\prime}$ as vertices called $c_{11}^{\prime}, c_{21}^{\prime}, c_{12}^{\prime}$, and $c_{22}^{\prime}$, respectively. Denote $G^{*}=G^{*}\left(U_{1}^{*}, U_{2}^{*}\right)$ as the resulting graph and let $c_{11}^{\prime} c_{21}^{\prime}, c_{12}^{\prime} c_{22}^{\prime} \in E\left(G^{*}\right)$. Denote $M^{\prime}:=M-$ $\left\{a_{11}^{\prime} a_{21}^{\prime}, b_{11}^{\prime} b_{21}^{\prime}, a_{12}^{\prime} a_{22}^{\prime}, b_{12}^{\prime} b_{22}^{\prime}\right\} \cup\left\{c_{11}^{\prime} c_{21}^{\prime}, c_{12}^{\prime} c_{22}^{\prime}\right\}$. Define an auxiliary graph $H^{\prime}$ on $M^{\prime}$ as follows. If $x y, u v \in M^{\prime}-\left\{c_{11}^{\prime} c_{21}^{\prime}, c_{12}^{\prime} c_{22}^{\prime}\right\}$ with $x, u \in U_{1}^{\prime}$ then $x y \sim_{H^{\prime}} u v$ if and only if $x \sim_{G^{\prime}} v$ and $y \sim_{G^{\prime}} u$. For any $p q \in M^{\prime}-\left\{c_{11}^{\prime} c_{21}^{\prime}, c_{12}^{\prime} c_{22}^{\prime}\right\}$ with $p \in U_{2}^{\prime}, p q \sim_{H^{\prime}} c_{11}^{\prime} c_{21}^{\prime}$ (resp. $\left.p q \sim_{H^{\prime}} c_{12}^{\prime} c_{22}^{\prime}\right)$ if and only if $p \sim_{G^{\prime}} a_{11}^{\prime}, b_{11}^{\prime}$ and $q \sim_{G^{\prime}} a_{21}^{\prime}, b_{21}^{\prime}$ (resp. $p \sim_{G^{\prime}} a_{12}^{\prime}, b_{12}^{\prime}$ and $q \sim_{G^{\prime}} a_{22}^{\prime}, b_{22}^{\prime}$ ). Notice that there is a natural one-to-one correspondence between ladders with rungs in $M^{\prime}$ and paths in $H^{\prime}$. Since $\delta_{G^{*}}\left(U_{1}^{*}, U_{2}^{*}\right), \delta_{G^{*}}\left(U_{2}^{*}, U_{1}^{*}\right) \geq 3 m^{\prime} / 4+1$, we get $\delta\left(H^{\prime}\right) \geq m^{\prime} / 2+1$. Hence $H^{\prime}$ has a hamiltonian path starting with $x_{1}^{\prime} x_{2}^{\prime}$, ending with $y_{1}^{\prime} y_{2}^{\prime}$, and having $c_{11}^{\prime} c_{21}^{\prime}$ and $c_{12}^{\prime} c_{22}^{\prime}$ as two internal vertices. The path with the vertex $c_{11}^{\prime} c_{21}^{\prime}$ replaced by $a_{11}^{\prime} a_{21}^{\prime}$ and $b_{11}^{\prime} b_{21}^{\prime}$, and with the vertex $c_{12}^{\prime} c_{22}^{\prime}$ replaced by $a_{12}^{\prime} a_{22}^{\prime}$ and $b_{12}^{\prime} b_{22}^{\prime}$ is corresponding to the required ladders in $G^{\prime \prime}$.

If $T \in\left\{T_{1}, T_{2}\right\}$, then

$$
H=x_{1} x_{2} Q_{1} L_{1} Q_{2} L_{2} Q_{3} y_{1} y_{2} \cup T
$$

is a spanning Halin subgraph of $G$. Suppose now that $T \in\left\{T_{3}, T_{4}, T_{5}\right\}$ and $z$ is the pendent vertex. Then $z \in V_{1}^{\prime} \cup V_{2}^{\prime}-W$ by Claim 3.2.3. Suppose, w.l.o.g., that $z \in V_{2}^{\prime}-W$. Then by (iii) of Claim 3.2.3 and $\delta\left(V_{2}^{\prime}, V_{1}^{\prime}\right) \geq\left(1 / 2-2 \alpha_{1} / 3\right) n$ from (1), we have that $\operatorname{deg}_{G}\left(z, U_{1}^{\prime}\right) \geq$ $\operatorname{deg}_{G}\left(z, V_{1}^{\prime}-V\left(L_{1} \cup L_{2}\right)-V(T)\right) \geq\left(1-\alpha_{1}-10 \alpha_{2}\right) m>m / 2+1$. So $z$ has a neighbor on each side of the ladder $Q_{1} L_{1} Q_{2} L_{2} Q_{3}$, which has $m$ vertices on each side, and each side has at most $m / 2+1$ vertices from each partition of $U_{1}^{\prime}$ and $U_{2}^{\prime}$. Let $H^{\prime}$ be obtained from $x_{1} x_{2} Q_{1} L_{1} Q_{2} L_{2} Q_{3} y_{1} y_{2} \cup T$ by suppressing the degree 2 vertex $z$. Then $H^{\prime}$ is a Halin graph such that there exists one side of $Q_{1} L_{1} Q_{2} L_{2} Q_{3}$ with each vertex on it as a degree 3 vertex on a underlying tree of $H^{\prime}$. Let $z^{\prime}$ be a neighbor of $z$ such that $z^{\prime}$ has degree 3 in the underlying tree of $H^{\prime}$. Then

$$
H=x_{1} x_{2} Q_{1} L_{1} Q_{2} L_{2} Q_{3} y_{1} y_{2} \cup T \cup\left\{z z^{\prime}\right\},
$$

is a spanning Halin subgraph of $G$.

\subsection{Proof of Theorem 3.3}

In this section, we prove Theorem 3.3. In the first subsection, we introduce the Regularity Lemma, the Blow-up Lemma, and some related results. Then we show that $G$ contains a subgraph $T$ isomorphic to $T_{1}$ if $n$ is even and to $T_{2}$ if $n$ is odd. By showing that $G-V(T)$ contains a spanning ladder $L$ with its first rung adjacent to the head link of $T$ and its last rung adjacent to the tail link of $T$, we get a spanning Halin subgraph $H$ of $G$ formed by $L \cup T$.

\subsubsection{The Regularity Lemma and the Blow-up Lemma}

For any two disjoint non-empty vertex-sets $A$ and $B$ of a graph $G$, the density of $A$ and $B$ is the ratio $d(A, B):=\frac{e(A, B)}{|A| \cdot|B|}$. Let $\varepsilon$ and $\delta$ be two positive real numbers. The pair $(A, B)$ is called $\varepsilon$-regular if for every $X \subseteq A$ and $Y \subseteq B$ with $|X|>\varepsilon|A|$ and $|Y|>\varepsilon|B|,|d(X, Y)-d(A, B)|<\varepsilon$ holds. In addition, if $\delta(A, B)>\delta|B|$ and $\delta(B, A)>\delta|A|$, we say $(A, B)$ an $(\varepsilon, \delta)$-super regular pair. 
Lemma 3.4 (Regularity lemma-Degree form [25]) For every $\varepsilon>0$ there is an $M=M(\varepsilon)$ such that if $G$ is any graph with $n$ vertices and $d \in[0,1]$ is any real number, then there is a partition of the vertex-set $V(G)$ into $l+1$ clusters $V_{0}, V_{1}, \cdots, V_{l}$, and there is a spanning subgraph $G^{\prime} \subseteq G$ with the following properties.

- $l \leq M$;

- $\left|V_{0}\right| \leq \varepsilon n$, all clusters $\left|V_{i}\right|=\left|V_{j}\right| \leq\lceil\varepsilon n\rceil$ for all $1 \leq i \neq j \leq l$;

- $\operatorname{deg}_{G^{\prime}}(v)>\operatorname{deg}_{G}(v)-(d+\varepsilon) n$ for all $v \in V(G)$;

- $e\left(G^{\prime}\left[V_{i}\right]\right)=0$ for all $i \geq 1$;

- in $G^{\prime}$, all pairs $\left(V_{i}, V_{j}\right)(1 \leq i \neq j \leq l)$ are $\varepsilon$-regular, each with a density either 0 or greater than d.

Lemma 3.5 (Blow-up lemma [18]) For every $\delta, \Delta, c>0$, there exists an $\varepsilon=\varepsilon(\delta, \Delta, c)$ and $\gamma=\gamma(\delta, \Delta, c)>0$ such that the following holds. Let $(X, Y)$ be an $(\varepsilon, \delta)$-super-regular pair with $|X|=|Y|=N$. If a bipartite graph $H$ with $\Delta(H) \leq \Delta$ can be embedded in $K_{N, N}$ by a function $\phi$, then $H$ can be embedded in $(X, Y)$. Moreover, in each $\phi^{-1}(X)$ and $\phi^{-1}(Y)$ (the inverse image of $X$ and $Y$, respectively), fix at most $\gamma N$ special vertices $z$, each of which is equipped with a subset $S_{z}$ of $X$ or $Y$ of size at least $c N$. The embedding of $H$ into $(X, Y)$ exists even if we restrict the image of $z$ to be $S_{z}$ for all special vertices $z$.

Besides the above two lemmas, we also need the two lemmas below regarding regular pairs.

Lemma 3.6 If $(A, B)$ is an $\varepsilon$-regular pair with density $d$, then for any $A^{\prime} \subseteq A$ with $\left|A^{\prime}\right| \geq \varepsilon|A|$, there are at most $\varepsilon|B|$ vertices $b \in B$ such that $\operatorname{deg}\left(b, A^{\prime}\right)<(d-\varepsilon)\left|A^{\prime}\right|$.

Lemma 3.7 (Slicing lemma) Let $(A, B)$ be an $\varepsilon$-regular pair with density $d$, and for some $\nu>\varepsilon$, let $A^{\prime} \subseteq A$ and $B^{\prime} \subseteq B$ with $\left|A^{\prime}\right| \geq \nu|A|,\left|B^{\prime}\right| \geq \nu|B|$. Then $\left(A^{\prime}, B^{\prime}\right)$ is an $\varepsilon^{\prime}$-regular pair of density $d^{\prime}$, where $\varepsilon^{\prime}=\max \{\varepsilon / \nu, 2 \varepsilon\}$ and $d^{\prime}>d-\varepsilon$.

\subsubsection{Finding subgraph $T$}

Claim 3.3.1 Let $n$ be a sufficient large integer and $G$ an $n$-vertex graph with $\delta(G) \geq(n+1) / 2$. If $\Delta(G) \leq n-2$, then $G$ contains a subgraph $T$ isomorphic to $T_{1}$ if $n$ is even and to $T_{2}$ if $n$ is odd.

Proof. (The proof can be much easier if uses the assumption that $G$ is not in Extremal Case 2, but we show it here just using the conditions on minimum and maximum degrees.) Suppose first that $n$ is even. As $\Delta(G) \leq n-2, G$ has two vertices $x_{1}$ and $y$ such that $x_{1} y \notin$ $E(G)$. Since $\delta(G) \geq(n+1) / 2$, there exists $x \in \Gamma\left(x_{1}, y\right)$. If $G$ has no independent set of size $n / 2-1$, we can find $x_{2} \in \Gamma\left(x_{1}, x\right)-\{y\}$ and $y_{1}, y_{2} \in \Gamma(y)-\left\{x, x_{1}, x_{2}\right\}$ such that $y_{1} y_{2} \in E(G)$. Hence $G\left[\left\{x, y, x_{1}, x_{2}, y_{1}, y_{2}\right\}\right]$ contains a subgraph $T$ isomorphic to $T_{1}$. So we assume $G$ has an independent set $S$ of size $n / 2-1$. Then $\delta(G-S) \geq 2$ and $\delta(S, V(G)-S)=|V(G)|-|S|$. Let $x_{1} y_{1}$ and $y y_{2}$ be two independent edges in $G-S$, and $x, y_{1}$ be any two distinct vertices in $S$. Then $x, y_{1} \in \Gamma\left(x_{1}, y_{1}, y, y_{2}\right)$ and $G\left[\left\{x, y, x_{1}, x_{2}, y_{1}, y_{2}\right\}\right]$ contains a subgraph $T$ isomorphic to $T_{1}$.

Then assume that $n$ is odd. Assume first that $G$ has no independent set of size $(n+1) / 2-4$. We show in the first step that $G$ contains a subgraph isomorphic to $K_{4}^{-}\left(K_{4}\right.$ with one edge removed). Let $y z \in E(G)$. As $\delta(G) \geq(n+1) / 2$, there exists $y_{1} \in \Gamma(y, z)$. If there exists $y_{2} \in \Gamma(y, z)-\left\{y_{1}\right\}$, we are done. Otherwise, $\left(\Gamma(y)-\left\{y_{1}, z\right\}\right) \cap\left(\Gamma(z)-\left\{y_{1}, y\right\}\right)=\emptyset$. As $\delta(G) \geq(n+1) / 2, y_{1}$ is adjacent to a vertex $y_{2} \in \Gamma(y) \cup \Gamma(z)-\left\{y_{1}, y, z\right\}$. Assume $y_{2} \in$ 
$\Gamma(z)-\left\{y_{1}, y\right\}$. Then $G\left[\left\{y, y_{1}, z, y_{2}\right\}\right]$ contains a copy of $K_{4}^{-}$. Choose $x \in \Gamma(y)-\left\{z, y_{1}, y_{2}\right\}$ and choose an edge $x_{1} x_{2} \in G\left[\Gamma(x)-\left\{y, y_{1}, y_{2}, z\right\}\right]$. Then $G\left[\left\{y, y_{1}, z, y_{2}, x, x_{1}, x_{2}\right\}\right]$ contains a subgraph $T$ isomorphic to $T_{2}$. Let $S$ be a maximum independent set of $G$. So we have $|S| \geq(n+1) / 2-4$. Let $x, y_{1}, z \in S$. Then there exist an edge $x_{1} x_{2}$ such that $x_{1}, x_{2} \in \Gamma(x, z)$, and $y \in \Gamma\left(x, y_{1}\right)-\left\{x_{1}, x_{2}, z\right\}$. If $|S|<(n+1) / 2-2$, we can find $y_{2} \in \Gamma\left(y, y_{1}\right)-\left\{x, x_{1}, x_{2}, z\right\}$. Again, $G\left[\left\{y, y_{1}, z, y_{2}, x, x_{1}, x_{2}\right\}\right]$ contains a subgraph $T$ isomorphic to $T_{2}$. So $|S| \geq(n+1) / 2-2$. Assume $G-S$ does not contain any independent set of size at least $(n+1) / 2-6$. For any $u, v, w \in S, \Gamma(u, v, w, V(G)-S) \geq(n+1) / 2-4$ by Lemma 3.3. Let $x, y_{1}, z \in S$. As $G-S$ does not contain any independent set of size at least $(n+1) / 2-6$, there exist independent edges $x_{1} x_{2}, y y_{2}$ such that $x_{1}, x_{2}, y, y_{2} \in \Gamma\left(x, z, y_{1}, V(G)-S\right)$. Then $G\left[\left\{y, y_{1}, z, y_{2}, x, x_{1}, x_{2}\right\}\right]$ contains a subgraph $T$ isomorphic to $T_{2}$. So we assume that $G-S$ contains an independent set of size at least $(n+1) / 2-6$. We take $Q$ to be a maximum one. As $\delta(G-S) \geq 2$ and $Q$ is independent, there exist two vertices $x_{1}, y \in V(G)-S-Q$ such that both of them have at least $(n+1) / 13$ neighbors in $Q$. Note that $\delta(S, V(G)-S) \geq(n+1) / 2 \geq|V(G)-S|-1$ and $S$ is a maximum independent set in $G$. So there exist $x, z \in \Gamma\left(x_{1}, S\right)$ and $y_{1} \in \Gamma(y, S)-\{x, z\}$. As both $x_{1}$ and $y$ have at least $(n+1) / 13$ neighbors in $Q$ and $\delta(S, V(G)-S) \geq(n+1) / 2 \geq|V(G)-S|-1$, there exist $x_{2} \in \Gamma\left(x, x_{1}, z, Q\right)$ and $y_{2} \in \Gamma\left(y, y_{1}, Q\right)-\left\{x_{2}\right\}$. So $G\left[\left\{y, y_{1}, z, y_{2}, x, x_{1}, x_{2}\right\}\right]$ contains a subgraph $T$ isomorphic to $T_{2}$.

Let $T$ be a subgraph of $G$ as given by Claim 3.3.1. Suppose the head link of $T$ is $x_{1} x_{2}$ and the tail link of $T$ is $y_{1} y_{2}$. Let $G^{\prime}=G-V(T)$. We show in next section that $G^{\prime}$ contains a spanning ladder with its first rung being adjacent to $x_{1} x_{2}$ and its last rung being adjacent to $y_{1} y_{2}$. Let $n^{\prime}=\left|V\left(G^{\prime}\right)\right|$. Then we have $\delta\left(G^{\prime}\right) \geq(n+1) / 2-7 \geq n^{\prime} / 2-4 \geq(1 / 2-\beta) n^{\prime}$, where $\beta$ is the parameter defined in the two extremal cases.

\subsubsection{Finding a spanning ladder of $G^{\prime}$ with prescribed end rungs}

Theorem 3.4 Let $n^{\prime}$ be a sufficiently large even integer and $G^{\prime}$ an $n^{\prime}$-vertex subgraph of $G$ obtained by removing vertices in $T$, where $T \in\left\{T_{1}, T_{2}\right\}$ has head link $x_{1} x_{2}$ and tail link $y_{1} y_{2}$. Suppose that $\delta\left(G^{\prime}\right) \geq(1 / 2-\beta) n^{\prime}$ and $G=G\left[V\left(G^{\prime}\right) \cup V(T)\right]$ is in Non-extremal Case, then $G^{\prime}$ contains a spanning ladder with its first rung adjacent to $x_{1} x_{2}$ and its last rung adjacent to $y_{1} y_{2}$.

Proof. We fix the following sequence of parameters

$$
0<\varepsilon \ll d \ll \beta \ll 1
$$

and specify their dependence as the proof proceeds.

Let $\beta$ be the parameter defined in the two extremal cases. Then we choose $d \ll \beta$ and choose

$$
\varepsilon=\frac{1}{4} \epsilon(d / 2,3, d / 4)
$$

following the definition of $\epsilon$ in the Blow-up Lemma.

Applying the Regularity Lemma to $G^{\prime}$ with parameters $\varepsilon$ and $d$, we obtain a partition of $V\left(G^{\prime}\right)$ into $l+1$ clusters $V_{0}, V_{1}, \cdots, V_{l}$ for some $l \leq M \leq M(\varepsilon)$, and a spanning subgraph $G^{\prime \prime}$ of $G^{\prime}$ with all described properties in the Regularity Lemma. In particular, for all $v \in V\left(G^{\prime}\right)$,

$$
\operatorname{deg}_{G^{\prime \prime}}(v)>\operatorname{deg}_{G^{\prime}}(v)-(d+\varepsilon) n^{\prime} \geq(1 / 2-\beta-\varepsilon-d) n^{\prime} \geq(1 / 2-2 \beta) n^{\prime}
$$

provided that $\varepsilon+d \leq \beta$. On the other hand,

$$
e\left(G^{\prime \prime}\right) \geq e\left(G^{\prime}\right)-\frac{(d+\varepsilon)}{2}\left(n^{\prime}\right)^{2}>e\left(G^{\prime}\right)-d\left(n^{\prime}\right)^{2}
$$


by $\varepsilon<d$.

We further assume that $l=2 k$ is even; otherwise, we eliminate the last cluster $V_{l}$ by removing all the vertices in this cluster to $V_{0}$. As a result, $\left|V_{0}\right| \leq 2 \varepsilon n^{\prime}$, and

$$
(1-2 \varepsilon) n^{\prime} \leq l N=2 k N \leq n^{\prime},
$$

where $N=\left|V_{i}\right|$ for $1 \leq i \leq l$.

For each pair $i$ and $j$ with $1 \leq i \neq j \leq l$, we write $V_{i} \sim V_{j}$ if $d\left(V_{i}, V_{j}\right) \geq d$. As in other applications of the Regularity Lemma, we consider the reduced graph $G_{r}$, whose vertex set is $\{1,2, \cdots, l\}$ and two vertices $i$ and $j$ are adjacent if and only if $V_{i} \sim V_{j}$. From $\delta\left(G^{\prime \prime}\right)>$ $(1 / 2-2 \beta) n^{\prime}$, we claim that $\delta\left(G_{r}\right) \geq(1 / 2-2 \beta) l$. Suppose not, and let $i_{0} \in V\left(G_{r}\right)$ be a vertex with $\operatorname{deg}_{G r}\left(i_{0}\right)<(1 / 2-2 \beta) l$. Let $V_{i_{0}}$ be the cluster in $G$ corresponding to $i_{0}$. Then we have

$$
(1 / 2-\beta) n^{\prime}\left|V_{i_{0}}\right| \leq\left|E_{G^{\prime}}\left(V_{i_{0}}, V-V_{i_{0}}\right)\right|<(1 / 2-2 \beta) l N\left|V_{i_{0}}\right|+2 \varepsilon n^{\prime}\left|V_{i_{0}}\right|<(1 / 2-\beta) n^{\prime}\left|V_{i_{0}}\right| .
$$

This gives a contradiction by $l N \leq n^{\prime}$ from inequality (4).

Let $A$ be a cluster of $G^{\prime \prime}$. We say $A$ is an $(\varepsilon, d)$-cluster if for any distinct cluster $B$ of $G^{\prime \prime}$ with $d(A, B)>0,(A, B)$ is an $\varepsilon$-regular pair with density at least $d$. Let $x \in V\left(G^{\prime}\right)$ be a vertex and $A$ an $(\varepsilon, d)$-cluster. We say $x$ is typical to $A$ if $\operatorname{deg}(x, A) \geq(d-\varepsilon)|A|$, and in this case, we write $x \sim A$.

Claim 3.3.2 Each vertex in $\left\{x_{1}, x_{2}, y_{1}, y_{2}\right\}$ is typical to at least $(1 / 2-2 \beta) l$ clusters in $\left\{V_{1}, \cdots, V_{l}\right\}$.

Proof. Suppose on the contrary that there exists $x \in\left\{x_{1}, x_{2}, y_{2}, y_{2}\right\}$ such that $x$ is typical to less than $(1 / 2-2 \beta) l$ clusters in $\left\{V_{1}, \cdots, V_{l}\right\}$. Then we have $\operatorname{deg}_{G^{\prime}}(x)<(1 / 2-2 \beta) l N+(d+$ $\varepsilon) n^{\prime} \leq(1 / 2-\beta) n^{\prime}$ by $l N \leq n^{\prime}$ and $d+\varepsilon \leq \beta$.

Let $x \in V\left(G^{\prime}\right)$ be a vertex. Denote by $\mathcal{V}_{x}$ the set of clusters to which $x$ typical.

Claim 3.3.3 There exist $V_{x_{1}} \in \mathcal{V}_{x_{1}}$ and $V_{x_{2}} \in \mathcal{V}_{x_{2}}$ such that $d\left(V_{x_{1}}, V_{x_{2}}\right) \geq d$.

Proof. We show the claim by considering two cases based on the size of $\left|\mathcal{V}_{x_{1}} \cap \mathcal{V}_{x_{2}}\right|$.

Case 1. $\left|\mathcal{V}_{x_{1}} \cap \mathcal{V}_{x_{2}}\right| \leq 2 \beta l$.

Then we have $\left|\mathcal{V}_{x_{1}}-\mathcal{V}_{x_{2}}\right| \geq(1 / 2-4 \beta) l$ and $\left|\mathcal{V}_{x_{2}}-\mathcal{V}_{x_{1}}\right| \geq(1 / 2-4 \beta) l$. We conclude that there is an edge between $\mathcal{V}_{x_{1}}-\mathcal{V}_{x_{2}}$ and $\mathcal{V}_{x_{2}}-\mathcal{V}_{x_{1}}$ in $G_{r}$. For otherwise, let $\mathcal{U}$ be the union of clusters in $\mathcal{V}_{x_{1}} \cap \mathcal{V}_{x_{2}}, W=V_{0} \cup \mathcal{U} \cup V(T)$. Let $V_{1}$ be the set of vertices contained in clusters in $\mathcal{V}_{x_{1}}-\mathcal{V}_{x_{2}}$, and $V_{2}$ be the set of vertices contained in clusters in $\mathcal{V}_{x_{1}}-\mathcal{V}_{x_{2}}$. Then $V_{1}$ and $V_{2}$ is a partition of $V(G)-W$. Furthermore, $|W| \leq 5 \beta n, e\left(V_{1}, V_{2}\right) \leq(d+\varepsilon) n^{\prime}\left|V_{1}\right| \leq(d+\varepsilon) n^{\prime}(1+4 \beta) l N \leq \beta n^{2}$, and $\delta\left(G\left[V_{i}\right]\right) \geq \delta(G)-7-|W|-(d+\varepsilon) n^{\prime} \geq \delta(G)-|W|-\beta n$. These imply that $W$ is an approximate vertex-cut of parameter $\beta$ with size at most $5 \beta n$, implying that $G$ is in Extremal Case 1.

Case 2. $\left|\mathcal{V}_{x_{1}} \cap \mathcal{V}_{x_{2}}\right|>2 \beta l$.

We may assume that $\mathcal{V}_{x_{1}} \cap \mathcal{V}_{x_{2}}$ is an independent set in $G_{r}$. For otherwise, we are done by finding an edge within $\mathcal{V}_{x_{1}} \cap \mathcal{V}_{x_{2}}$. Also we may assume that $E_{G_{r}}\left(\mathcal{V}_{x_{1}} \cap \mathcal{V}_{x_{2}}, \mathcal{V}_{x_{1}}-\mathcal{V}_{x_{2}}\right)=\emptyset$ and $E_{G_{r}}\left(\mathcal{V}_{x_{1}} \cap \mathcal{V}_{x_{2}}, \mathcal{V}_{x_{2}}-\mathcal{V}_{x_{1}}\right)=\emptyset$. Since $\delta\left(G_{r}\right) \geq(1 / 2-2 \beta) l$ and $\delta_{G_{r}}\left(\mathcal{V}_{x_{1}} \cap \mathcal{V}_{x_{2}}, \mathcal{V}_{x_{1}} \cup \mathcal{V}_{x_{2}}\right)=0$, we know that $l-\left|\mathcal{V}_{x_{1}} \cup \mathcal{V}_{x_{2}}\right| \geq(1 / 2-2 \beta) l$. Hence, $\left|\mathcal{V}_{x_{1}} \cup \mathcal{V}_{x_{2}}\right|=\left|\mathcal{V}_{x_{1}}\right|+\left|\mathcal{V}_{x_{2}}\right|-\left|\mathcal{V}_{x_{1}} \cap \mathcal{V}_{x_{2}}\right| \leq(1 / 2+2 \beta) l$. This gives that $\left|\mathcal{V}_{x_{1}} \cap \mathcal{V}_{x_{2}}\right| \geq\left|\mathcal{V}_{x_{1}}\right|+\left|\mathcal{V}_{x_{2}}\right|-(1 / 2+2 \beta) l \geq(1 / 2-2 \beta) l+(1 / 2-2 \beta) l-(1 / 2+$ $2 \beta) l \geq(1 / 2-6 \beta) l$. Let $\mathcal{U}$ be the union of clusters in $\mathcal{V}_{x_{1}} \cap \mathcal{V}_{x_{2}}$. Then $|\mathcal{U}| \geq(1 / 2-7 \beta) n$ and $\Delta(G[\mathcal{U}]) \leq(d+\varepsilon) n^{\prime} \leq \beta n$. This shows that $G$ is in Extremal Case 2.

Similarly, we have the following claim: 
Claim 3.3.4 There exist $V_{y_{1}} \in \mathcal{V}_{y_{1}}-\left\{V_{x_{1}}, V_{x_{2}}\right\}$ and $V_{y_{2}} \in \mathcal{V}_{y_{2}}-\left\{V_{x_{1}}, V_{x_{2}}\right\}$ such that $d\left(V_{y_{1}}, V_{y_{2}}\right) \geq$ $d$.

Claim 3.3.5 The reduced graph $G_{r}$ has a hamiltonian path $X_{1} Y_{1} \cdots X_{k} Y_{k}$ such that $\left\{X_{1}, Y_{1}\right\}=$ $\left\{V_{x_{1}}, V_{x_{2}}\right\}$ and $\left\{X_{k}, Y_{k}\right\}=\left\{V_{y_{1}}, V_{y_{2}}\right\}$.

Proof. We contract the edges $V_{x_{1}} V_{x_{2}}$ and $V_{y_{1}} V_{y_{2}}$ in $G_{r}$. Denote the two new vertices as $V_{x}^{\prime}$ and $V_{y}^{\prime}$ respectively, and denote the resulting graph as $G_{r}^{\prime}$. Then we show that $G_{r}^{\prime}$ contains a hamiltonian $\left(V_{x}^{\prime}, V_{y}^{\prime}\right)$-path. This path is corresponding to a required hamiltonian path in $G_{r}$.

To show $G_{r}^{\prime}$ has a hamiltonian $\left(V_{x}^{\prime}, V_{y}^{\prime}\right)$-path, we need the following generalized version of a result due to Nash-Williams [21] : Let $Q$ be a 2-connected graph of order $m$. If $\delta(Q) \geq$ $\max \{(m+2) / 3+1, \alpha(Q)+1\}$, then $Q$ is hamiltonian connected, where $\alpha(Q)$ is the size of a largest independent set of $Q$.

We claim that $G_{r}^{\prime}$ is $2 \beta l$-connected. Otherwise, let $S$ be a vertex-cut of $G_{r}^{\prime}$ with $|S|<2 \beta l$ and $\mathcal{S}$ the vertex set corresponding to $S$ in $G$. Since $\delta\left(G_{r}^{\prime}\right) \geq(1 / 2-2 \beta) l-2$ and $|S|<2 \beta l$, we know that $G_{r}^{\prime}-S$ has exactly two components. Let $W=\mathcal{S} \cup V_{0} \cup V(T), V_{1}$ the set of vertices contained in clusters corresponding to vertices in one component of $G_{r}^{\prime}-S$, and $V_{2}=V(G)-V_{1}-W$. Then it is easy to check that $e\left(V_{1}, V_{2}\right) \leq \beta n^{2}$ and $\delta\left(G\left[V_{i}\right]\right) \geq \delta(G)-|W|-\beta n$. Hence $W$ is an approximate vertex-cut of parameter $\beta$ with size at most $5 \beta n$, showing that $G$ is in Extremal Case 1. Since $n^{\prime}=N l+\left|V_{0}\right| \leq(l+2) \varepsilon n^{\prime}$, we have that $l \geq 1 / \varepsilon-2 \geq 1 / \beta$. Hence, $G_{r}^{\prime}$ is 2-connected. As $G$ is not in Extremal Case $2, \alpha\left(G_{r}^{\prime}\right) \leq(1 / 2-7 \beta) l$. By $\delta\left(G_{r}\right) \geq(1 / 2-2 \beta) l$, we have $\delta\left(G_{r}^{\prime}\right) \geq(1 / 2-2 \beta) l-2 \geq \max \{(l+2) / 3+1,(1 / 2-7 \beta) l+1\}$. Thus, by the result on hamiltonian connectedness given above, we know that $G_{r}^{\prime}$ contains a hamiltonian $\left(V_{x}^{\prime}, V_{y}^{\prime}\right)$-path.

Claim 3.3.6 For each $1 \leq i \leq k$, there exist $X_{i}^{\prime} \subseteq X_{i}$ and $Y_{i}^{\prime} \subseteq Y_{i}$ such that each of the following holds:

(1) $\left|X_{1}^{\prime}\right| \geq(1-\varepsilon)\left|X_{1}\right|-1,\left|Y_{k}^{\prime}\right| \geq(1-\varepsilon)\left|Y_{k}\right|-1,\left|Y_{1}^{\prime}\right| \geq(1-\varepsilon)\left|Y_{1}\right|,\left|X_{k}^{\prime}\right| \geq(1-\varepsilon)\left|X_{k}\right|$, and $\left|X_{i}^{\prime}\right| \geq(1-\varepsilon)\left|X_{i}\right|, 2 \leq i \leq k-1 ;$

(2) $\left(X_{i}^{\prime}, Y_{i}^{\prime}\right)$ is $(2 \varepsilon, d-3 \varepsilon)$-super-regular with density at least $d-\varepsilon$;

(3) $\left|Y_{1}^{\prime}\right|=\left|X_{1}^{\prime}\right|+1,\left|X_{k}^{\prime}\right|=\left|Y_{k}^{\prime}\right|+1$, and $\left|X_{i}^{\prime}\right|=\left|Y_{i}^{\prime}\right|, 2 \leq i \leq k-1$; and

(4) for any $A, B \in\left\{X_{1}^{\prime}, Y_{1}^{\prime}, \cdots, X_{k}^{\prime}, Y_{k}^{\prime}\right\}$, if $d(A, B)>0$, then $(A, B)$ is $2 \varepsilon$-regular with density at least $d-\varepsilon$. Consequently, each $A$ is a $(2 \varepsilon, d-\varepsilon)$ cluster.

Proof. For each $1 \leq i \leq k$, let

$$
\begin{aligned}
X_{i}^{\prime \prime} & =\left\{x \in X_{i} \mid \operatorname{deg}\left(x, Y_{i}\right) \geq(d-\varepsilon) N\right\}, \text { and } \\
Y_{i}^{\prime \prime} & =\left\{y \in Y_{i} \mid \operatorname{deg}\left(y, X_{i}\right) \geq(d-\varepsilon) N\right\} .
\end{aligned}
$$

If necessary, we either take a subset $X_{i}^{\prime}$ of $X_{i}^{\prime \prime}$ or take a subset $Y_{i}^{\prime}$ of $Y_{i}^{\prime \prime}$ such that $\left|Y_{1}^{\prime}\right|=\left|X_{1}^{\prime}\right|+1$, $\left|X_{k}^{\prime}\right|=\left|Y_{k}^{\prime}\right|+1$, and $\left|X_{i}^{\prime}\right|=\left|Y_{i}^{\prime}\right|$ for $2 \leq i \leq k-1$. Since $\left(X_{i}, Y_{i}\right)$ is $\varepsilon$-regular, we have $\left|X_{i}^{\prime \prime}\right|,\left|Y_{i}^{\prime \prime}\right| \geq(1-\varepsilon) N$. This gives that $\left|X_{1}^{\prime}\right|,\left|Y_{k}^{\prime}\right| \geq(1-\varepsilon) N-1,\left|Y_{1}^{\prime}\right| \geq(1-\varepsilon) N,\left|X_{k}^{\prime}\right| \geq(1-\varepsilon) N$, and $\left|X_{i}^{\prime}\right|=\left|Y_{i}^{\prime}\right| \geq(1-\varepsilon) N$ for $2 \leq i \leq k-1$. As a result, we have $\operatorname{deg}\left(x, Y_{i}^{\prime}\right) \geq(d-2 \varepsilon) N$ for each $x \in X_{i}^{\prime}$ and $\operatorname{deg}\left(y, X_{i}^{\prime}\right) \geq(d-2 \varepsilon) N-1 \geq(d-3 \varepsilon) N$ for each $y \in Y_{i}^{\prime}$. By the Slicing lemma (Lemma 3.7), $\left(X_{i}^{\prime}, Y_{i}^{\prime}\right)$ is $2 \varepsilon$-regular with density at least $d-\varepsilon$. Hence $\left(X_{i}^{\prime}, Y_{i}^{\prime}\right)$ is $(2 \varepsilon, d-3 \varepsilon)$-super-regular for each $1 \leq i \leq k$. The last assertion is again an application of the Slicing lemma. 
For $1 \leq i \leq k$, we call each $X_{i}^{\prime}, Y_{i}^{\prime}$ a super-regularized cluster(sr-cluster), and call $X_{i}^{\prime}$ and $Y_{i}^{\prime}$ partners of each other and write $P\left(X_{i}^{\prime}\right)=Y_{i}^{\prime}$ and $P\left(Y_{i}^{\prime}\right)=X_{i}^{\prime}$. Denote $R=V_{0} \cup$ $\left(\bigcup_{i=1}^{k}\left(\left(X_{i} \cup Y_{i}\right)-\left(X_{i}^{\prime} \cup Y_{i}^{\prime}\right)\right)\right)$. Since $\left|\left(X_{i} \cup Y_{i}\right)-\left(X_{i}^{\prime} \cup Y_{i}^{\prime}\right)\right| \leq 2 \varepsilon N$ for $2 \leq i \leq k-1$ and $\left|\left(X_{1} \cup Y_{1}\right)-\left(X_{1}^{\prime} \cup Y_{1}^{\prime}\right)\right|,\left|\left(X_{k} \cup Y_{k}\right)-\left(X_{k}^{\prime} \cup Y_{k}^{\prime}\right)\right| \leq 2 \varepsilon N+1$, we have $|R| \leq 2 \varepsilon n+2 k \varepsilon N+2 \leq 3 \varepsilon n^{\prime}$. As $n^{\prime}$ is even and $\left|X_{1}^{\prime}\right|+\left|Y_{1}^{\prime}\right|+\cdots+\left|X_{k}^{\prime}\right|+\left|Y_{k}^{\prime}\right|$ is even, we know $|R|$ is even. We arbitrarily group vertices in $R$ into $|R| / 2$ pairs. Given two vertices $u, v \in R$, we define a $(u, v)$-chain of length $2 t$ as distinct sr-clusters $A_{1}, B_{1}, \cdots, A_{t}, B_{t}$ such that $u \sim A_{1} \sim B_{1} \sim \cdots \sim A_{t} \sim B_{t} \sim v$ and each $A_{j}$ and $B_{j}$ are partners, in other words, $\left\{A_{j}, B_{j}\right\}=\left\{X_{i_{j}}^{\prime}, Y_{i_{j}}^{\prime}\right\}$ for some $i_{j} \in\{1, \cdots, k\}$. Recall here $u \sim A_{1}$ means that $\operatorname{deg}\left(u, A_{1}\right) \geq(d-3 \varepsilon)\left|A_{1}\right|$, and $A_{1} \sim B_{1}$ means that the two vertices corresponding to $A_{1}$ and $B_{1}$ are adjacent in $G_{r}$. We call such a chain of length $2 t$ a $2 t$-chain.

Claim 3.3.7 For each pair $(u, v)$ in $R$, we can find $a(u, v)$-chain of length at most 4 such that every sr-cluster is contained in at most $d^{2} N / 5$ chains.

Proof. Suppose we have found chains for the first $m<2 \varepsilon n^{\prime}$ pairs of vertices in $R$ such that no sr-cluster is contained in more than $d^{2} N / 5$ chains. Let $\Omega$ be the set of all sr-clusters that are contained exactly in $d^{2} N / 5$ chains. Then

$$
\frac{d^{2} N}{5}|\Omega| \leq 4 m<8 \varepsilon n^{\prime} \leq 8 \varepsilon \frac{2 k N}{1-2 \varepsilon},
$$

where the last inequality follows from (4). Therefore,

$$
|\Omega| \leq \frac{80 k \varepsilon}{d^{2}(1-2 \varepsilon)} \leq \frac{80 l \varepsilon}{d^{2}} \leq \beta l / 2
$$

provided that $1-2 \varepsilon \geq 1 / 2$ and $80 \varepsilon \leq d^{2} \beta / 2$.

Consider now a pair $(w, z)$ of vertices in $R$ which does not have a chain found so far, we want to find a $(w, z)$-chain using sr-clusters not in $\Omega$. Let $\mathcal{U}$ be the set of all sr-clusters to which $w$ typical but not in $\Omega$, and let $\mathcal{V}$ be the set of all sr-clusters to which $z$ typical but not in $\Omega$. We claim that $|\mathcal{U}|,|\mathcal{V}| \geq(1 / 2-2 \beta) l$. To see this, we first observe that any vertex $x \in R$ is typical to at least $(1 / 2-3 \beta / 2) l$ sr-clusters. For instead,

$$
\begin{aligned}
(1 / 2-\beta) n^{\prime} & \leq \operatorname{deg}_{G^{\prime}}(x)<(1 / 2-3 \beta / 2) l N+(d-3 \varepsilon) l N+3 \varepsilon n^{\prime}, \\
& \leq(1 / 2-3 \beta / 2+d) n^{\prime} \\
& \left.<(1 / 2-\beta) n^{\prime} \text { (provided that } d<\beta / 2\right),
\end{aligned}
$$

showing a contradiction. Since $|\Omega| \leq \beta l / 2$, we have $|\mathcal{U}|,|\mathcal{V}| \geq(1 / 2-2 \beta) l$. Let $P(\mathcal{U})$ and $P(\mathcal{V})$ be the set of the partners of clusters in $\mathcal{U}$ and $\mathcal{V}$, respectively. By the definition of the chains, a cluster $A \in \Omega$ if and only its partner $P(A) \in \Omega$. Hence, $(P(\mathcal{U}) \cup P(\mathcal{V})) \cap \Omega=\emptyset$. Notice also that each cluster has a unique partner, and so we have $|P(\mathcal{U})|=|\mathcal{U}| \geq(1 / 2-2 \beta) l$ and $|P(\mathcal{V})|=|\mathcal{V}| \geq(1 / 2-2 \beta) l$.

If $E_{G_{r}}(P(\mathcal{U}), P(\mathcal{V})) \neq \emptyset$, then there exist two adjacent clusters $B_{1} \in P(\mathcal{U}), A_{2} \in P(\mathcal{V})$. If $B_{1}$ and $A_{2}$ are partners of each other, then $w \sim A_{2} \sim B_{1} \sim z$ gives a $(w, z)$-chain of length 2 . Otherwise, assume $A_{1}=P\left(B_{1}\right)$ and $B_{2}=P\left(A_{2}\right)$, then $w \sim A_{1} \sim B_{1} \sim A_{2} \sim B_{2} \sim z$ gives a $(w, z)$-chain of length 4 . Hence we assume that $E_{G_{r}}(P(\mathcal{U}), P(\mathcal{V}))=\emptyset$. We may assume that $P(\mathcal{U}) \cap P(\mathcal{V}) \neq \emptyset$. Otherwise, let $\mathcal{S}$ be the union of clusters contained in $V\left(G_{r}\right)-(P(\mathcal{U}) \cup P(\mathcal{V}))$. Then $\mathcal{S} \cup R \cup V(T)$ with $|\mathcal{S} \cup R \cup V(T)| \leq 4 \beta n^{\prime}+3 \varepsilon n^{\prime}+7 \leq 5 \beta n$ (provided that $3 \varepsilon+7 / n^{\prime}<\beta$ ) is an approximate vertex-cut of $G$, implying that $G$ is in Extremal Case 1. As $E_{G_{r}}(P(\mathcal{U}), P(\mathcal{V}))=\emptyset$, any cluster in $P(\mathcal{U}) \cap P(\mathcal{V})$ is adjacent to at least $(1 / 2-2 \beta) l$ clusters in $V\left(G_{r}\right)-(P(\mathcal{U}) \cup P(\mathcal{V}))$ 
by $\delta\left(G_{r}\right) \geq(1 / 2-2 \beta) l$. This implies that $|P(\mathcal{U}) \cup P(\mathcal{V})| \leq(1 / 2+2 \beta) l$, and thus $|P(\mathcal{U}) \cap P(\mathcal{V})| \geq$ $|P(\mathcal{U})|+|P(\mathcal{V})|-|P(\mathcal{U}) \cup P(\mathcal{V})| \geq(1 / 2-6 \beta) l$. Then $P(\mathcal{U}) \cap P(\mathcal{V})$ is corresponding to a subset $V_{1}$ of $V(G)$ such that $\left|V_{1}\right| \geq(1 / 2-6 \beta) l N \geq(1 / 2-7 \beta) n$ and $\Delta\left(G\left[V_{1}\right]\right) \leq(d+\varepsilon) n^{\prime} \leq \beta n$. This implies that $G$ is in Extremal Case 2, showing a contradiction.

By Claim 3.3.7, each vertex in $R$ is contained in a unique chain of length at most 4 . Let $Z$ be an sr-cluster, and $u \in R$ be a vertex. We say $u$ and $Z$ are chain-adjacent to each other if in the chain which contains $u, Z$ appears next to $u$. For each sr-cluster $Z \in\left\{X_{1}^{\prime}, Y_{1}^{\prime}, \cdots, X_{k}^{\prime}, Y_{k}^{\prime}\right\}$, let $R(Z)$ denote the set of vertices in $R$ such that each of the vertices is chain-adjacent to $Z$. Let $R_{4}(Z)=\{u \in R(Z) \mid u$ is contained in a 4-chain $\}$, and let $S_{4}(Z)$ denote the set of sr-clusters distinct from $Z$ such that each of them is adjacent to the partner $P(Z)$ of $Z$ in a 4-chain which contains $Z$. That is, for each $A \in S_{4}(Z)$, there exists $u \in R_{4}(Z)$ and $v \in R-R_{4}(Z)$ such that $u \sim Z \sim P(Z) \sim A \sim P(A) \sim v$ is a 4-chain. If $Z \in\left\{X_{1}^{\prime}, \cdots, X_{k}^{\prime}\right\}$, then for each sr-cluster $A \in S_{4}(Z)$, let $c(A)$ denote the number of 4-chains which contains $Z \sim P(Z) \sim A \sim P(A)$ as a sequence. For each $A \in S_{4}(Z)$, choose $c(A)$ vertices in $A$ such that each of them has at least $(d-3 \varepsilon)|Z|>3 d^{2} N / 5$ neighbors in $P(Z)$. (Since $(P(Z), A)$ is $2 \varepsilon$-regular with density at least $d-\varepsilon$, we know that there are at least $(1-2 \varepsilon)|A|$ vertices in $A$ with this property by Lemma 3.6.) Let $R^{\prime}(Z)$ be the union of $R(Z)$ and the set of vertices chosen from $A \in S_{4}(P(Z))$ above, and let

$$
\omega(A)=\sum_{A \in S_{4}(Z),} c(A) .
$$

Note that by the definitions, $R^{\prime}(Z)$ is only defined for sr-clusters $Z \in\left\{Y_{1}^{\prime}, \cdots, Y_{k}^{\prime}\right\}$, and $\omega(A)$ is defined only for sr-clusters $A \in\left\{X_{1}^{\prime}, \cdots, X_{k}^{\prime}\right\}$.

Claim 3.3.8 For each $i=1,2, \cdots, k$, each of the following holds.

(a) $\left|R\left(X_{i}^{\prime}\right)\right| \leq d^{2} N / 5$ and $\left|R^{\prime}\left(Y_{i}^{\prime}\right)\right| \leq d^{2} N / 5$.

(b) $\left|R\left(X_{i}^{\prime}\right)-R_{4}\left(X_{i}^{\prime}\right)\right|=\left|R\left(Y_{i}^{\prime}\right)-R_{4}\left(Y_{i}^{\prime}\right)\right|$.

(c) $\omega\left(X_{i}^{\prime}\right)=\left|R_{4}\left(Y_{i}^{\prime}\right)\right|$.

(d) $\left|R^{\prime}\left(Y_{i}^{\prime}\right)-R\left(Y_{i}^{\prime}\right)\right|=\left|R_{4}\left(X_{i}^{\prime}\right)\right|$.

Proof. By Claim 3.3.7, each sr-cluster is contained in at most $d^{2} N / 5$ chains, and a chain contains $X_{i}^{\prime}$ if and only if it also contains $Y_{i}^{\prime}$ by its definition. Since both $\left|R\left(X_{i}^{\prime}\right)\right|$ and $\left|R^{\prime}\left(Y_{i}^{\prime}\right)\right|$ are bounded above by the number of chains which contain them, we have that $\left|R\left(X_{i}^{\prime}\right)\right| \leq d^{2} N / 5$ and $\left|R^{\prime}\left(Y_{i}^{\prime}\right)\right| \leq d^{2} N / 5$. By the definition of 2-chains, a vertex in $R$ is chain-adjacent to an sr-cluster $A$ in a 2-chain if and only if there exists another vertex in $R$ which is chain-adjacent to the partner $P(A)$ of $A$. Thus $\left|R\left(X_{i}^{\prime}\right)-R_{4}\left(X_{i}^{\prime}\right)\right|=\left|R\left(Y_{i}^{\prime}\right)-R_{4}\left(Y_{i}^{\prime}\right)\right|$. By the definition, if $X_{i}^{\prime} \in S_{4}(Z)$ for some sr-cluster $Z$, then $c\left(X_{i}^{\prime}\right)$ is the number of 4-chains which contains $Y_{i}^{\prime} \sim X_{i}^{\prime} \sim P(Z) \sim Z$ as a sequence. All of such 4-chains is just the set of 4-chains in which $Y_{i}^{\prime}$ is chain-adjacent to a vertex in $R$. Since each vertex in $R$ is contained in a unique chain, we then have that $\omega\left(X_{i}^{\prime}\right)=\left|R_{4}\left(Y_{i}^{\prime}\right)\right|$. Since each vertex in $R^{\prime}\left(Y_{i}^{\prime}\right)-R\left(Y_{i}^{\prime}\right)$ is corresponding to a 4-chain in which $X_{i}^{\prime}$ is chain-adjacent to a vertex in $R$, we have that $\left|R^{\prime}\left(Y_{i}^{\prime}\right)-R\left(Y_{i}^{\prime}\right)\right|=\left|R_{4}\left(X_{i}^{\prime}\right)\right|$.

Claim 3.3.9 For each $i=1,2, \cdots, k$, there exist vertex-disjoint ladders $L_{x}^{i}$, $L_{y}^{i}$ such that

(a) $R\left(X_{i}^{\prime}\right) \subseteq V\left(L_{x}^{i}\right) \subseteq R\left(X_{i}^{\prime}\right) \cup X_{i}^{\prime} \cup Y_{i}^{\prime}$ and $R^{\prime}\left(Y_{i}^{\prime}\right) \subseteq V\left(L_{y}^{i}\right) \subseteq X_{i}^{\prime} \cup Y_{i}^{\prime} \cup R^{\prime}\left(Y_{i}^{\prime}\right)$;

(b) $\left|\left(V\left(L_{x}^{i}\right) \cup V\left(L_{y}^{i}\right)\right) \cap X_{i}^{\prime}\right|=4\left|R\left(X_{i}^{\prime}\right)\right|+3\left|R\left(Y_{i}^{\prime}\right)\right|+3\left|R_{4}\left(X_{i}^{\prime}\right)\right|-2$ and $\left|\left(V\left(L_{x}^{i}\right) \cup V\left(L_{y}^{i}\right)\right) \cap Y_{i}^{\prime}\right|=$ $4\left|R\left(Y_{i}^{\prime}\right)\right|+4\left|R_{4}\left(X_{i}^{\prime}\right)\right|+3\left|R\left(X_{i}^{\prime}\right)\right|-2$; and

(c) the vertices on the first and last rungs of each of $L_{x}^{i}$ and $L_{y}^{i}$ are contained in $X_{i}^{\prime} \cup Y_{i}^{\prime}$. 
Proof. Notice that by Claim 3.3.6, $\left(X_{i}^{\prime}, Y_{i}^{\prime}\right)$ is $2 \varepsilon$-regular with density at least $d-\varepsilon$. Let $R\left(X_{i}^{\prime}\right)=\left\{x_{1}, \cdots, x_{r}\right\}$. For each $j, 1 \leq j \leq r$, since $\left|\Gamma\left(x_{j}, X_{i}^{\prime}\right)\right| \geq(d-3 \varepsilon)\left|X_{i}^{\prime}\right|>2 \varepsilon\left|X_{i}^{\prime}\right|$, by Lemma 3.6, there exists a vertex set $B_{j} \subseteq Y_{i}^{\prime}$ with $\left|B_{j}\right| \geq(1-2 \varepsilon)\left|Y_{i}^{\prime}\right|$ such that for each $b_{1} \in B_{j}$, $\operatorname{deg}\left(b_{1}, \Gamma\left(x_{j}, X_{i}^{\prime}\right)\right) \geq(d-3 \varepsilon)\left|\Gamma\left(x_{j}, X_{i}^{\prime}\right)\right|>4\left|R\left(X_{i}^{\prime}\right)\right|$. If $r \geq 2$, for $j=1, \cdots, r-1$, by Lemma 3.6, there also exists a vertex set $B_{j, j+1} \subseteq Y_{i}^{\prime}$ with $\left|B_{j, j+1}\right| \geq(1-4 \varepsilon)\left|Y_{i}^{\prime}\right|$ such that for each $b_{2} \in$ $B_{j, j+1}$, we have $\operatorname{deg}\left(b_{2}, \Gamma\left(x_{j}, X_{i}^{\prime}\right)\right) \geq(d-3 \varepsilon)\left|\Gamma\left(x_{j}, X_{i}^{\prime}\right)\right|>4\left|R\left(X_{i}^{\prime}\right)\right|$ and $\operatorname{deg}\left(b_{2}, \Gamma\left(x_{j+1}, X_{i}^{\prime}\right)\right) \geq$ $(d-3 \varepsilon)\left|\Gamma\left(x_{j+1}, X_{i}^{\prime}\right)\right|>4\left|R\left(X_{i}^{\prime}\right)\right|$. When $r \geq 2$, since $\left|B_{j}\right|,\left|B_{j, j+1}\right|,\left|B_{j+1}\right| \geq(d-3 \varepsilon)\left|Y_{i}^{\prime}\right|>2 \varepsilon\left|Y_{i}^{\prime}\right|$, there is a set $A \subseteq X_{i}^{\prime}$ with $|A| \geq(1-6 \varepsilon)\left|X_{i}^{\prime}\right| \geq\left|R\left(X_{i}^{\prime}\right)\right|$ such that for each $a \in A, \operatorname{deg}\left(a, B_{j}\right) \geq$ $(d-3 \varepsilon)\left|B_{j}\right|, \operatorname{deg}\left(a, B_{j, j+1}\right) \geq(d-3 \varepsilon)\left|B_{j, j+1}\right|$ and $\operatorname{deg}\left(a, B_{j+1}\right) \geq(d-3 \varepsilon)\left|B_{j+1}\right|$. Notice that $(d-3 \varepsilon)\left|B_{j}\right|,(d-3 \varepsilon)\left|B_{j, j+1}\right|,(d-3 \varepsilon)\left|B_{j+1}\right| \geq(d-3 \varepsilon)(1-4 \varepsilon)\left|Y_{i}^{\prime}\right|>3\left|R\left(X_{i}^{\prime}\right)\right|$. Hence we can choose distinct vertices $u_{1}, u_{2}, \cdots, u_{r-1} \in A$ such that $\operatorname{deg}\left(u_{j}, B_{j}\right), \operatorname{deg}\left(u_{j}, B_{j, j+1}\right), \operatorname{deg}\left(u_{j}, B_{j+1}\right) \geq$ $3\left|R\left(X_{i}^{\prime}\right)\right|$. Then we can choose distinct vertices $y_{23}^{j} \in \Gamma\left(u_{j}, B_{j}\right), z_{j} \in \Gamma\left(u_{j}, B_{j, j+1}\right)$ and $y_{12}^{j+1} \in$ $\Gamma\left(u_{j}, B_{j+1}\right)$ for each $j$, and choose distinct and unchosen vertices $y_{12}^{1} \in B_{1}$ and $y_{23}^{r} \in B_{r}$. Finally, as for each vertex $b_{1} \in B_{j}$, we have $\operatorname{deg}\left(b_{1}, \Gamma\left(x_{j}, X_{i}^{\prime}\right)\right)>4\left|R\left(X_{i}^{\prime}\right)\right|$ and for each vertex $b_{2} \in$ $B_{j, j+1}$, we have $\operatorname{deg}\left(b_{2}, \Gamma\left(x_{j}, X_{i}^{\prime}\right)\right), \operatorname{deg}\left(b_{2}, \Gamma\left(x_{j+1}, X_{i}^{\prime}\right)\right)>4\left|R\left(X_{i}^{\prime}\right)\right|$, we can choose $x_{j 1}, x_{j 2}, x_{j 3} \in$ $\Gamma\left(x_{j}, X_{i}^{\prime}\right)-\left\{u_{1}, \cdots, u_{r-1}\right\}$ such that $y_{12}^{j} \in \Gamma\left(x_{j 1}, x_{j 2}, Y_{i}^{\prime}\right), y_{23}^{j} \in \Gamma\left(x_{j 2}, x_{j 3}, Y_{i}^{\prime}\right)$, and $z_{j} \in$ $\Gamma\left(x_{i 3}, x_{i+1,1}, Y_{i}^{\prime}\right)$. (When $i \geq 2$, we choose all these vertices such that they are not used by existing ladders. The possibility of doing this is guaranteed by the degree conditions and the small sizes of the existing ladders.) Let $L_{x}^{i}$ be the graph with

$$
V\left(L_{x}^{i}\right)=R\left(X_{i}^{\prime}\right) \cup\left\{x_{i 1}, x_{i 2}, x_{i 3}, y_{12}^{i}, y_{23}^{i}, z_{i}, u_{i}, x_{r 1}, x_{r 2}, x_{r 3}, y_{12}^{r}, y_{23}^{r} \mid 1 \leq i \leq r-1\right\} \quad \text { and }
$$

$E\left(L_{x}^{i}\right)$ consisting of the edges $x_{r} x_{r 1}, x_{r} x_{r 2}, x_{r} x_{r 3}, y_{12}^{r} x_{r 1}, y_{12}^{r} x_{r 2}, y_{23}^{r} x_{r 2}, y_{23}^{r} x_{r 3}$ and the edges indicated below for each $1 \leq i \leq r-1$ :

$$
x_{i} \sim x_{i 1}, x_{i 2}, x_{i 3} ; y_{12}^{i} \sim x_{i 1}, x_{i 2} ; y_{23}^{i} \sim x_{i 2}, x_{i 3} ; z_{i} \sim x_{i 3}, x_{i+1,1} ; u_{i} \sim x_{i 3}, x_{i+1,1}, z_{i} .
$$

It is easy to check that $L_{x}^{i}$ is a ladder spanning on $R\left(X_{i}^{\prime}\right), 4\left|R\left(X_{i}^{\prime}\right)\right|-1$ vertices from $X_{i}^{\prime}$ and $3\left|R\left(X_{i}^{\prime}\right)\right|-1$ vertices from $Y_{i}^{\prime}$. Similarly, we can find a ladder $L_{y}^{i}$ spanning on $R^{\prime}\left(Y_{i}^{\prime}\right), 4\left|R^{\prime}\left(Y_{i}^{\prime}\right)\right|-1$ vertices from $Y_{i}^{\prime}$ and $3\left|R^{\prime}\left(Y_{i}^{\prime}\right)\right|-1$ vertices from $X_{i}^{\prime}$. The constructions of ladders $L_{x}^{i}$ and $L_{y}^{i}$ verify both of statements (a) and (c). The statement (b) is seen by the construction of the ladders and (d) of Claim 3.3.8 which says that $\left|R^{\prime}\left(Y_{i}^{\prime}\right)\right|=\left|R\left(Y_{i}^{\prime}\right)\right|+\left|R_{4}\left(X_{i}^{\prime}\right)\right|$.

For each $i=1,2, \cdots, k-1$, let $X_{i}^{* *}=X_{i}^{\prime}-V\left(\bigcup_{i=1}^{k}\left(L_{x}^{i} \cup L_{y}^{i}\right)\right)$ and $Y_{i}^{* *}=Y_{i}^{\prime}-V\left(\bigcup_{i=1}^{k}\left(L_{x}^{i} \cup\right.\right.$ $\left.L_{y}^{i}\right)$ ). Using Lemma 3.6, for $i \in\{1, \cdots, k-1\}$, choose $y_{i}^{*} \in Y_{i}^{* *}$ such that $\left|A_{i+1}\right| \geq d N / 4$, where $A_{i+1}:=X_{i+1}^{* *} \cap \Gamma\left(y_{i}^{*}\right)$. This is possible, as $\left(Y_{i}^{* *}, X_{i+1}^{* *}\right)$ is $4 \varepsilon$-regular with density at least $d-3 \varepsilon$. (Applying Slicing lemma based on $\left(Y_{i}^{\prime}, X_{i+1}^{\prime}\right)$ ). Similarly, choose $x_{i+1}^{*} \in A_{i+1}$ such that $\left|D_{i}\right| \geq d N / 4$, where $D_{i}:=Y_{i}^{* *} \cap \Gamma\left(x_{i+1}^{*}\right)$. Let $S=\left\{y_{i}^{*}, x_{i+1}^{*} \mid 1 \leq i \leq k-1\right\}$, and let $X_{i}^{*}=X_{i}^{* *}-S$ and $Y_{i}^{*}=Y_{i}^{* *}-S$. We have the following holds.

Claim 3.3.10 For each $i=1,2, \cdots, k,\left|X_{i}^{*}\right|=\left|Y_{i}^{*}\right|$ and $\left(X_{i}^{*}, Y_{i}^{*}\right)$ is $(4 \varepsilon, d / 2)$-super-regular.

Proof. We show that $\left|X_{i}^{*}\right|=\left|Y_{i}^{*}\right|$ for each $i, 1 \leq i \leq k$. Since $\left|Y_{1}^{\prime}\right|=\left|X_{1}^{\prime}\right|+1,\left|X_{k}^{\prime}\right|=$ $\left|Y_{k}^{\prime}\right|+1$, and $\left|X_{i}^{\prime}\right|=\left|Y_{i}^{\prime}\right|$ for $2 \leq i \leq k-1$, and $\left|X_{1}^{* *}\right|=\left|X_{1}^{*}\right|,\left|Y_{k}^{* *}\right|=\left|Y_{k}^{*}\right|$, and $\left|X_{i}^{* *}\right|=\left|X_{i}^{*}\right|-1$, $\left|Y_{j}^{* *}\right|=\left|Y_{j}^{*}\right|-1$ for $2 \leq i \leq k, 1 \leq j \leq k-1$, it suffices to show that $\left|X_{i}^{\prime} \cap V\left(\bigcup_{i=1}^{k}\left(L_{x}^{i} \cup L_{y}^{i}\right)\right)\right|=$ $\left|Y_{i}^{\prime} \cap V\left(\bigcup_{i=1}^{k}\left(L_{x}^{i} \cup L_{y}^{i}\right)\right)\right|$. This is clear by (b) of Claim 3.3.9 and Claim 3.3.8. As

$$
\begin{aligned}
& \left|X_{i}^{\prime} \cap V\left(\bigcup_{i=1}^{k}\left(L_{x}^{i} \cup L_{y}^{i}\right)\right)\right|=4\left|R\left(X_{i}^{\prime}\right)\right|+3\left|R\left(Y_{i}^{\prime}\right)\right|+3\left|R_{4}\left(X_{i}^{\prime}\right)\right|-2+\omega\left(X_{i}^{\prime}\right) \\
= & 4\left|R\left(X_{i}^{\prime}\right)-R_{4}\left(X_{i}^{\prime}\right)\right|+3\left|R\left(Y_{i}^{\prime}\right)-R_{4}\left(Y_{i}^{\prime}\right)\right|+7\left|R_{4}\left(X_{i}^{\prime}\right)\right|+3\left|R_{4}\left(Y_{i}^{\prime}\right)\right|-2+\omega\left(X_{i}^{\prime}\right) \\
= & 7\left|R\left(X_{i}^{\prime}\right)-R_{4}\left(X_{i}^{\prime}\right)\right|+7\left|R_{4}\left(X_{i}^{\prime}\right)\right|+4\left|R_{4}\left(Y_{i}^{\prime}\right)\right|-2,
\end{aligned}
$$


and

$$
\begin{aligned}
& \left|Y_{i}^{\prime} \cap V\left(\bigcup_{i=1}^{k}\left(L_{x}^{i} \cup L_{y}^{i}\right)\right)\right|=3\left|R\left(X_{i}^{\prime}\right)\right|+4\left|R\left(Y_{i}^{\prime}\right)\right|+4\left|R_{4}\left(X_{i}^{\prime}\right)\right|-2 \\
= & 3\left|R\left(X_{i}^{\prime}\right)-R_{4}\left(X_{i}^{\prime}\right)\right|+4\left|R\left(Y_{i}^{\prime}\right)-R_{4}\left(Y_{i}^{\prime}\right)\right|+7\left|R_{4}\left(X_{i}^{\prime}\right)\right|+4\left|R_{4}\left(Y_{i}^{\prime}\right)\right|-2 \\
= & 7\left|R\left(X_{i}^{\prime}\right)-R_{4}\left(X_{i}^{\prime}\right)\right|+7\left|R_{4}\left(X_{i}^{\prime}\right)\right|+4\left|R_{4}\left(Y_{i}^{\prime}\right)\right|-2 .
\end{aligned}
$$

Since $\left|R\left(X_{i}^{\prime}\right)\right|,\left|R^{\prime}\left(Y_{i}^{\prime}\right)\right| \leq d^{2} N / 5$ for each $i$, by the first part of argument, $\mid X_{i}^{\prime} \cap V\left(\bigcup_{i=1}^{k}\left(L_{x}^{i} \cup\right.\right.$ $\left.\left.L_{y}^{i}\right)\right)|\leq 4| R\left(X_{i}^{\prime}\right)|+4| R^{\prime}\left(Y_{i}^{\prime}\right) \mid-2 \leq 2 d^{2} N-2$ and $\left|Y_{i}^{\prime} \cap V\left(\bigcup_{i=1}^{k}\left(L_{x}^{i} \cup L_{y}^{i}\right)\right)\right| \leq 4\left|R\left(X_{i}^{\prime}\right)\right|+$ $4\left|R^{\prime}\left(Y_{i}^{\prime}\right)\right|-2 \leq 2 d^{2} N-2$. Thus $\left|X_{i}^{*}\right|,\left|Y_{i}^{*}\right| \geq\left(1-\varepsilon-2 d^{2}\right) N$. As $\varepsilon, d \ll 1$, we can assume that $1-\varepsilon-2 d^{2}<1 / 2$. Thus, by Slicing lemma based on the $2 \varepsilon$-regular pair $\left(X_{i}^{\prime}, Y_{i}^{\prime}\right)$, we know that $\left(X_{i}^{*}, Y_{i}^{*}\right)$ is $4 \varepsilon$-regular. Recall from Claim 3.3.6 that $\left(X_{i}^{\prime}, Y_{i}^{\prime}\right)$ is $(2 \varepsilon, d-3 \varepsilon)$-super-regular, we know that for each $x \in X_{i}^{*}, \operatorname{deg}\left(x, Y_{i}^{*}\right) \geq\left(d-3 \varepsilon-2 d^{2}\right)\left|Y_{i}^{*}\right|>d\left|Y_{i}^{*}\right| / 2$. Similarly, we have for each $y \in Y_{i}^{*}, \operatorname{deg}\left(y, X_{i}^{*}\right) \geq d\left|X_{i}^{*}\right| / 2$. Thus $\left(X_{i}^{*}, Y_{i}^{*}\right)$ is $(4 \varepsilon, d / 2)$-super-regular.

For each $i=1,2, \cdots, k-1$, now set $B_{i+1}:=Y_{i+1}^{*} \cap \Gamma\left(x_{i+1}^{*}\right)$ and $C_{i}:=X_{i}^{*} \cap \Gamma\left(y_{i}^{*}\right)$. Since $\left(X_{i}^{*}, Y_{i}^{*}\right)$ is $(4 \varepsilon, d / 2)$-super-regular, we have $\left|B_{i}\right|,\left|C_{i}\right| \geq d\left|X_{i}^{*}\right| / 2>d\left|X_{i}^{*}\right| / 4$. Recall from Claim 3.3.5 that $\left\{X_{1}, Y_{1}\right\}=\left\{V_{x_{1}}, V_{x_{2}}\right\}$ and $\left\{X_{k}, Y_{k}\right\}=\left\{V_{y_{1}}, V_{y_{2}}\right\}$. We assume, w.l.o.g., that $X_{1}=V_{x_{1}}$ and $X_{k}=V_{y_{1}}$. Let $A_{1}=X_{1}^{*} \cap \Gamma\left(x_{1}\right), B_{1}=Y_{1}^{*} \cap \Gamma\left(x_{2}\right), C_{k}=X_{k}^{*} \cap \Gamma\left(y_{1}\right)$, and $D_{k}=$ $Y_{k}^{*} \cap \Gamma\left(y_{2}\right)$. Since $\operatorname{deg}\left(x_{1}, X_{1}\right) \geq(d-\varepsilon) N$, we have $\operatorname{deg}\left(x_{1}, X_{1}^{*}\right) \geq\left(d-\varepsilon-2 \varepsilon-2 d^{2}\right) N \geq d\left|X_{1}^{*}\right| / 4$, and thus $\left|A_{1}\right| \geq d\left|X_{1}^{*}\right| / 4$. Similarly, we have $\left|B_{1}\right|,\left|C_{k}\right|,\left|D_{k}\right| \geq d\left|X_{1}^{*}\right| / 4$. For each $1 \leq i \leq k$, we assume that $L_{x}^{i}=a_{1}^{i} b_{1}^{i}-L_{x}^{i}-c_{1}^{i} d_{1}^{i}$ and $L_{y}^{i}=a_{2}^{i} b_{2}^{i}-L_{y}^{i}-c_{2}^{i} d_{2}^{i}$, where $a_{j}^{i}, c_{j}^{i} \in Y_{i}^{\prime} \subseteq Y_{i}$ and $b_{j}^{i}, d_{j}^{i} \in X_{i}^{\prime} \subseteq X_{i}$ for $j=1,2$. For $j=1,2$, let $A_{j}^{i}=X_{i}^{*} \cap \Gamma\left(a_{j}^{i}\right), C_{j}^{i}=X_{i}^{*} \cap \Gamma\left(c_{j}^{i}\right)$, $B_{j}^{i}=Y_{i}^{*} \cap \Gamma\left(b_{j}^{i}\right)$, and $D_{j}^{i}=Y_{i}^{*} \cap \Gamma\left(d_{j}^{i}\right)$. Since $\left(X_{i}^{\prime}, Y_{i}^{\prime}\right)$ is $(2 \varepsilon, d-3 \varepsilon)$-super-regular, for $j=1,2$, we have $\left|\Gamma\left(a_{j}^{i}, X_{i}^{\prime}\right)\right|,\left|\Gamma\left(c_{j}^{i}, X_{i}^{\prime}\right)\right| \geq(d-3 \varepsilon)\left|X_{i}^{\prime}\right|$ and $\left|\Gamma\left(b_{j}^{i}, Y_{i}^{\prime}\right)\right|,\left|\Gamma\left(d_{j}^{i}, Y_{i}^{\prime}\right)\right| \geq(d-3 \varepsilon)\left|Y_{i}^{\prime}\right|$. Thus, we have $\left|A_{j}^{i}\right|,\left|B_{j}^{i}\right|,\left|C_{j}^{i}\right|,\left|D_{j}^{i}\right| \geq(d-3 \varepsilon)\left|X_{i}^{\prime}\right|-2 d^{2} N \geq d\left|X_{i}^{*}\right| / 4=d\left|Y_{i}^{*}\right| / 4$.

We now apply the Blow-up lemma on $\left(X_{i}^{*}, Y_{i}^{*}\right)$ to find a spanning ladder $L^{i}$ with its first and last rungs being contained in $A_{i} \times B_{i}$ and $C_{i} \times D_{i}$, respectively, and for $j=1,2$, its $(2 j)$-th and $(2 j+1)$-th rungs being contained in $A_{j}^{i} \times B_{j}^{i}$ and $C_{j}^{i} \times D_{j}^{i}$, respectively. We can then insert $L_{x}^{i}$ between the 2 nd and 3 rd rungs of $L^{i}$ and $L_{y}^{i}$ between the 4th and 5 th rungs of $L^{i}$ to obtained a ladder $\mathcal{L}^{i}$ spanning on $X_{i} \cup Y_{i}-S$. Finally, $\mathcal{L}^{1} y_{1}^{*} x_{2}^{*} \mathcal{L}^{2} \cdots y_{k-1}^{*} x_{k}^{*} \mathcal{L}^{k}$ is a spanning ladder of $G^{\prime}$ with its first rung adjacent to $x_{1} x_{2}$ and its last rung adjacent to $y_{1} y_{2}$.

The proof is now complete.

\section{References}

[1] M. Aigner and S. Brandt. Embedding arbitrary graphs of maximum degree two. J. London Math. Soc. (2), 48(1):39-51, 1993.

[2] Michael O. Albertson, David M. Berman, Joan P. Hutchinson, and Carsten Thomassen. Graphs with homeomorphically irreducible spanning trees. J. Graph Theory, 14(2):247-258, 1990.

[3] C. A. Barefoot. Hamiltonian connectivity of the Halin graphs. Congr. Numer., 58:93-102, 1987. Eighteenth Southeastern International Conference on Combinatorics, Graph Theory, and Computing (Boca Raton, Fla., 1987).

[4] J. A. Bondy. Pancyclic graphs: recent results. In Infinite and finite sets (Colloq., Keszthely, 1973; dedicated to P. Erdös on his 60th birthday), Vol. I, pages 181-187. Colloq. Math. Soc. János Bolyai, Vol. 10. North-Holland, Amsterdam, 1975.

[5] J. A. Bondy and L. Lovász. Lengths of cycles in Halin graphs. J. Graph Theory, 9(3):397410, 1985. 
[6] Phong Châu. An Ore-type theorem on Hamiltonian square cycles. Graphs Combin., 29(4):795-834, 2013.

[7] G. Chen, H. Enomoto, K. Ozeki, and S. Tsuchiya. Plane triangulations without a spanning halin subgraph: Counterexamples to the lovsz-plummer conjecture on halin graphs. SIAM Journal on Discrete Mathematics, 29(3):1423-1426, 2015.

[8] G. Cornuejols, D. Naddef, and W. R. Pulleyblank. Halin graphs and the travelling salesman problem. Math. Programming, 26(3):287-294, 1983.

[9] A. Czygrinow and H. A. Kierstead. 2-factors in dense bipartite graphs. Discrete Math., 257(2-3):357-369, 2002. Kleitman and combinatorics: a celebration (Cambridge, MA, 1999).

[10] Andrzej Czygrinow, Louis DeBiasio, and H. A. Kierstead. 2-factors of bipartite graphs with asymmetric minimum degrees. SIAM J. Discrete Math., 24(2):486-504, 2010.

[11] G. A. Dirac. Some theorems on abstract graphs. Proc. London Math. Soc. (3), 2:69-81, 1952.

[12] P. Erdős. Problem 9. In Theory of Graphs and Its Applications, Proceedings of the Symposium held in Smolenice in June 1963 (Ed. M. Fiedler), page 159. Prague, Czechoslovakia: Publishing House of the Czechoslovak Academy of Sciences, 1964.

[13] Genghua Fan and H. A. Kierstead. Hamiltonian square-paths. J. Combin. Theory Ser. B, 67(2):167-182, 1996.

[14] Ronald J. Gould. Advances on the Hamiltonian problem-a survey. Graphs Combin., 19(1):7-52, 2003.

[15] R. L. Graham, M. Grötschel, and L. Lovász, editors. Handbook of combinatorics. Vol. 1, 2. Elsevier Science B.V., Amsterdam; MIT Press, Cambridge, MA, 1995.

[16] R. Halin. Studies on minimally $n$-connected graphs. In Combinatorial Mathematics and its Applications (Proc. Conf., Oxford, 1969), pages 129-136. Academic Press, London, 1971.

[17] S. B. Horton, R. Gary Parker, and Richard B. Borie. Corrigendum: "On some results pertaining to Halin graphs" [Congr. Numer. 89 (1992), 65-87; MR1208942 (93j:05129)]. In Proceedings of the Twenty-fourth Southeastern International Conference on Combinatorics, Graph Theory, and Computing (Boca Raton, FL, 1993), volume 93, page 5, 1993.

[18] G. N. Komlós, J.and Sárközy and E. Szemerédi. Blow-up lemma. Combinatorica, 17(1):109$123,1997$.

[19] János Komlós, Gábor N. Sárközy, and Endre Szemerédi. On the square of a Hamiltonian cycle in dense graphs. In Proceedings of the Seventh International Conference on Random Structures and Algorithms (Atlanta, GA, 1995), volume 9, pages 193-211, 1996.

[20] L. Lovász and M. D. Plummer. On a family of planar bicritical graphs. Proc. London Math. Soc. (3), 30:160-176, 1975.

[21] C. St. J. A. Nash-Williams. Edge-disjoint Hamiltonian circuits in graphs with vertices of large valency. In Studies in Pure Mathematics (Presented to Richard Rado), pages 157-183. Academic Press, London, 1971.

[22] M. Skowrońska. The pancyclicity of Halin graphs and their exterior contractions. In Cycles in graphs (Burnaby, B.C., 1982), volume 115 of North-Holland Math. Stud., pages 179-194. North-Holland, Amsterdam, 1985. 
[23] Z. Skupień. Crowned trees and planar highly Hamiltonian graphs. In Contemporary methods in graph theory, pages 537-555. Bibliographisches Inst., Mannheim, 1990.

[24] Katherine Staden and Andrew Treglown. On degree sequences forcing the square of a hamilton cycle, 2014.

[25] E. Szemerédi. Regular partitions of graphs. In Problèmes combinatoires et théorie des graphes (Colloq. Internat. CNRS, Univ. Orsay, Orsay, 1976), volume 260 of Colloq. Internat. CNRS, pages 399-401. CNRS, Paris, 1978. 